\title{
A community-driven resource for genomic epidemiology and antimicrobial resistance prediction of Neisseria gonorrhoeae at Pathogenwatch
}

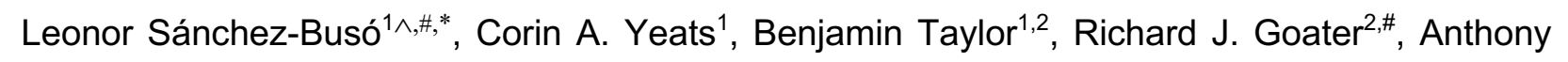
Underwood $^{2}$, Khalil Abudahab², Silvia Argimón², Kevin C. Ma ${ }^{3}$, Tatum D. Mortimer ${ }^{3}$, Daniel Golparian $^{4}$, Michelle J. Cole ${ }^{5, \wedge}$, Yonatan H. Grad ${ }^{3,6, \wedge}$, Irene Martin ${ }^{7, \wedge}$, Brian H. Raphael ${ }^{8, \wedge}$, William M. Shafer ${ }^{9,10, \wedge}$, Gianfranco Spiteri ${ }^{11, \wedge}$, Katy Town ${ }^{8, \wedge}$, Teodora Wi ${ }^{12, \wedge}$, Simon R. Harris ${ }^{13}$, Magnus Unemo $^{4, \wedge}$ and David M. Aanensen ${ }^{1,2, \wedge, *}$

${ }^{1}$ Centre for Genomic Pathogen Surveillance, Big Data Institute, Nuffield Department of Medicine, University of Oxford, Oxford, Oxfordshire, United Kingdom.

${ }^{2}$ Centre for Genomic Pathogen Surveillance, Wellcome Sanger Institute, Wellcome Genome Campus, Hinxton, Cambridgeshire, United Kingdom.

${ }^{3}$ Department of Immunology and Infectious Diseases, Harvard T. H. Chan School of Public Health, Boston, Massachusetts, United States of America.

${ }^{4}$ World Health Organization Collaborating Centre for Gonorrhoea and Other STIs, Department of Laboratory Medicine, Faculty of Medicine and Health, Örebro University, Örebro, Sweden.

${ }^{5}$ National Infection Service, Public Health England, London, United Kingdom.

${ }^{6}$ Division of Infectious Diseases, Department of Medicine, Brigham and Women's Hospital, Harvard Medical School, Boston, Massachusetts, United States of America.

${ }^{7}$ National Microbiology Laboratory, Public Health Agency of Canada, Winnipeg, Manitoba, Canada.

${ }^{8}$ Division of STD prevention, National Center for HIVIAIDS, Viral Hepatitis, STD and TB Prevention, Centers for Disease Control and Prevention, Atlanta, Georgia, United States of America.

${ }^{9}$ Department of Microbiology and Immunology and Emory Antibiotic Resistance Center, Emory University School of Medicine, Atlanta, Georgia, United States of America.

${ }^{10}$ Laboratories of Bacterial Pathogenesis, Veterans Affairs Medical Center, Decatur, Georgia, United States of America.

${ }^{11}$ European Centre for Disease Prevention and Control, Stockholm, Sweden.

12 Department of the Global HIV, Hepatitis and STI programmes, World Health Organization, Geneva, Switzerland.

${ }^{13}$ Microbiotica, Biodata Innovation Centre, Hinxton, Cambridgeshire, United Kingdom.

'Members of the N. gonorrhoeae Pathogenwatch Scientific Steering Group at the time of publication of this manuscript.

\#Current affiliations; Leonor Sánchez Busó: Genomics and Health Area, Foundation for the Promotion of Health and Biomedical Research in the Valencian Community (FISABIO-Public Health), Valencia, Spain; Richard J. Goater: European Molecular Biology Lab, Heidelberg, BadenWuerttemberg, Germany.

“Corresponding authors: Leonor Sánchez-Busó (leo.sanchez-buso@cgps.group) and David M. Aanensen (david.aanensen@cgps.group).

Keywords: Neisseria gonorrhoeae, Pathogenwatch, public health, genomics, epidemiology, surveillance, antimicrobial resistance. 


\section{Abstract}

49 Background: Antimicrobial resistant (AMR) Neisseria gonorrhoeae is an urgent threat to public health, as strains resistant to at least one of the two last line antibiotics used in empiric therapy of gonorrhoea, ceftriaxone and azithromycin, have spread internationally. Whole genome sequencing (WGS) data can be used to identify new AMR clones, transmission networks and inform the development of point-of-care tests for antimicrobial susceptibility, novel antimicrobials and vaccines. Community driven tools that provide an easy access to and analysis of genomic and epidemiological data is the way forward for public health surveillance.

Methods: Here we present a public health focussed scheme for genomic epidemiology of $N$. gonorrhoeae at Pathogenwatch (https://pathogen.watch/ngonorrhoeae). An international advisory group of experts in epidemiology, public health, genetics and genomics of $N$. gonorrhoeae was convened to inform on the utility of current and future analytics in the platform. We implement backwards compatibility with MLST, NG-MAST and NG-STAR typing schemes as well as an exhaustive library of genetic AMR determinants linked to a genotypic prediction of resistance to eight antibiotics. A collection of over 12,000 N. gonorrhoeae genome sequences from public archives has been quality-checked, assembled and made public together with available metadata for contextualization.

Results: AMR prediction from genome data revealed specificity values over $99 \%$ for azithromycin, ciprofloxacin and ceftriaxone and sensitivity values around $99 \%$ for benzylpenicillin and tetracycline. A case study using the Pathogenwatch collection of $N$. gonorrhoeae public genomes showed the global expansion of an azithromycin resistant lineage carrying a mosaic $\mathrm{mtr}$ over at least the last 10 years, emphasizing the power of Pathogenwatch to explore and evaluate genomic epidemiology questions of public health concern.

Conclusions: The N. gonorrhoeae scheme in Pathogenwatch provides customized bioinformatic pipelines guided by expert opinion that can be adapted to public health agencies and departments with little expertise in bioinformatics and lower resourced settings with internet connection but limited computational infrastructure. The advisory group will assess and identify ongoing public health needs in the field of gonorrhoea, particularly regarding gonococcal AMR, in order to further enhance utility with modified or new analytic methods. 


\section{Background}

83 Antimicrobial resistance (AMR) is an urgent threat to public health. Neisseria gonorrhoeae, the

84 strictly human pathogen causing the sexually-transmitted infection (STI) gonorrhoea, has developed or acquired resistance to the last-line antibiotics used in empiric therapy to treat the infection, and thus has become one of the major global priorities in order to tackle AMR. In 2017, due to the increase in AMR infections and the absence of an effective vaccine, the World Health Organization (WHO) included $N$. gonorrhoeae as a high priority pathogen in need of research and development of new antimicrobials and ideally a vaccine (1). In 2019, the Centers for Disease Control and Prevention (CDC) again included the gonococcus on the list of urgent threats in the United States (2). The most recent WHO estimates from 2016 indicate an annual global incidence of 87 million cases of gonorrhoea among adults $(3,4)$. Untreated cases can develop complications including an increased acquisition and transmission of HIV. In women, long-term infections can cause infertility, pelvic inflammatory disease, ectopic pregnancy, miscarriage or premature labour (5). Infections during pregnancy can transmit to newborns at birth causing eye damage that can have permanent effects on vision (6).

Strains of $N$. gonorrhoeae resistant to every recommended treatment have rapidly emerged, including resistance to penicillins, tetracyclines, fluoroquinolones, macrolides and the extendedspectrum cephalosporins (ESCs) (5-8). The current recommended treatment in many countries is a dual therapy with injectable ceftriaxone plus oral azithromycin, although reports of decreased susceptibility to ceftriaxone as well as azithromycin resistance have increased globally $(7,8)$. One case of failure of dual treatment was reported in 2016 in the United Kingdom (UK) (9). Additionally, in 2018 a gonococcal strain with resistance to ceftriaxone combined with high-level resistance to azithromycin was detected in both the UK and Australia (10). The transmission of a ceftriaxoneresistant clone (FC428) has been documented internationally since 2015 , raising concerns about the long-term effectiveness of the current treatment in the absence of an available alternative (11). In some countries such as in Japan, China and since 2019 in the UK, a single dose of 
resistance in N. gonorrhoeae and other STI pathogens such as Mycoplasma genitalium (12).

110 Extensive investigations have been ongoing for years to unveil the genetic mechanisms that 111 explain most of the observed susceptibility patterns for the main classes of antimicrobials for $N$.

112 gonorrhoeae. For ciprofloxacin, nearly all resistant strains have the GyrA S91F amino acid 113 alteration (13-15), however, resistance prediction from genomic data is not as straightforward for 114 other antibiotics. Known resistance mechanisms often involve additive or suppressive effects as well as epistatic interactions that all together explain just part of the observed phenotypic resistance. For example, there is good evidence that many mosaic structures of the penA gene are associated with decreased susceptibility to ESCs $(16,17)$, however, mosaics do not explain all cases of ESC resistance, especially for ceftriaxone, and some mosaic penA alleles do not cause decreased susceptibility or resistance to this antibiotic (16-19). On top of these, variants that overexpress the MtrCDE efflux pump, mutations in porB1b that reduce drug influx and nonmosaic mutations in penicillin-binding proteins also contribute to decreased susceptibility to ESCs (20). Furthermore, mutations in the $r p o B$ and $r p o D$ genes, encoding subunits of the RNA polymerase, have been recently related to resistance to ESCs in clinical $N$. gonorrhoeae isolates (21). Mutations in the 23S rRNA gene (A2045G and C2597T in N. gonorrhoeae nomenclature, coordinates from the WHO 2016 reference panel (22), A2059G and C2611T in Escherichia coli) are frequently associated with azithromycin resistance, as do variants in $m t r R$ or its promoter that increase the expression of the MtrCDE efflux pump (5). Recently, epistatic interactions between a mosaic $m t r$ promoter region and a mosaic $m t r D$ gene have also been reported to increase the expression of this pump, contributing to macrolide resistance $(23,24)$. Mutations in $r p / D$ have also been associated with reduced susceptibility to this antibiotic (25) and contrarily, loss-of-function mutations in mtrC have been linked to increased susceptibility to several antibiotics including azithromycin (26). Thus, we can relatively confidently predict decreased susceptibility or resistance to an antimicrobial using the current known genetic mechanisms, however, phenotypic testing is still necessary to detect resistant cases caused by unknown or novel mechanisms.

135 These inconsistencies with the genomic data will allow the discovery of these new mechanisms, which will keep improving the resistance predictions from WGS. 
137 A myriad of methods have been used to discriminate among strains of $N$. gonorrhoeae, from 138 phenotypic to DNA-based techniques (27), but whole genome sequencing (WGS) can provide 139 the complete genome information of a bacterial strain. The cost of amplifying all loci of the different 140 typing schemes via nucleic acid amplification and traditional Sanger sequencing can be more 141 expensive than the cost of WGS of one bacterial genome in many settings. With WGS, multiple 142 genetic AMR mechanisms as well as virulence and typing regions can be targeted simultaneously 143 with the appropriate bioinformatic tools and pipelines. It also provides a significant improvement 144 in resolution and accuracy over traditional molecular epidemiology and typing methods, allowing 145 a genome-wide comparison of strains that can: identify AMR clones, outbreaks, transmission 146 networks, national and international spread, known and novel resistance mechanisms as well as 147 also inform on the development of point-of-care tests for antimicrobial susceptibility, novel 148 antimicrobials and vaccines $(28,29)$. However, implementation of WGS for genomic surveillance 149 poses practical challenges, especially for Low- and Middle-Income Countries (LMICs), due to the need of a major investment to acquire and maintain the required infrastructure.

WGS produces a very high volume of data that needs to be pre-processed and analysed using bioinformatics. Bioinformatics expertise is not always readily available in laboratory and public health settings, and currently there are no international standards and proficiency trials for which algorithms to use to process WGS data. There are several open-source tools specialised in each step of the pipeline as well as proprietary software containing workflows that simplify the analyses. However, these are less customizable and may not be affordable for all $(30,31)$. Choosing the best algorithms and parameters when analysing genomic data is not straightforward as it requires a fair knowledge of the pathogen under study and its genome diversity. Multiple databases containing genetic determinants of AMR for bacterial pathogens are available $(30,31)$, however, choosing which one is most complete for a particular organism frequently requires an extensive

161 literature search. Public access web-based species-specific tools and AMR databases revised 162 and curated by experts would be the most approachable option for both well-resourced and LMICs 163 with a reliable internet connection. Very importantly though, the full benefits of using WGS for 
both molecular epidemiology and AMR prediction can only be achieved if the WGS data are linked

165 to phenotypic data for the gonococcal isolates and, as much as feasible, clinical and 166 epidemiological data for the patients.

167 Here, we present a public health focussed system to facilitate genomic epidemiology of $N$. gonorrhoeae within Pathogenwatch (https://pathogen.watch/ngonorrhoeae), which includes the latest analytics for typing, detection of genetic AMR determinants and prediction of AMR from $N$. gonorrhoeae genome data, linked to metadata where available, as well as a collection of over 12,000 gonococcal genomes from public archives for contextualization. We formed an advisory group including experts in the field of $N$. gonorrhoeae epidemiology, public health, AMR, genetics and genomics to consult on the development and design of the tool, such as the analytics and genetic AMR mechanisms to include, in order to adapt the platform for ongoing public health needs. We present this scheme as a community-steered model for genomic surveillance that can be applied to other pathogens.

\section{Methods}

\section{The Pathogenwatch platform: technical summary}

180 Pathogenwatch is a web-based platform with several different components. The main interface is 181 a React (32) single-page application with a style based on Material Design Lite (33). Phylogenetic 182 trees are plotted using Phylocanvas (34), maps using Leaflet (35) and networks with Sigma (36).

183 The back end is written in Node.js and contains an API service for the user interface and four 184 "Runner" services for the following analyses: species prediction, single-genome analyses, tree 185 building and core genome multi-locus sequence typing (cgMLST) clustering. Docker containers 186 are used for queuing tasks, streaming input or result files through standard input and storing 187 JSON data from standard output. A MongoDB cluster is used for data storage and task 188 queuing/synchronisation. Pathogenwatch shares some visualization components with Microreact 
189 (37), such as those associated with the phylogenetic tree and the map. However, Pathogenwatch 190 includes an analytical framework which is unique to this platform.

\section{Generation of the N. gonorrhoeae core genome library}

192 Pathogenwatch implements a library of core genome sequences for several supported organisms.

193 In the case of $N$. gonorrhoeae, a core gene set was built from the 14 finished reference genomes that constitute the $2016 \mathrm{WHO}$ reference strain panel (22) using the pangenome analysis tool Roary (38) as described in Harris et al (2018) (15). Briefly, the minimum percentage of identity for blastp was set to $97 \%$ and the resulting core genes were aligned individually using MAFFT. The resulting genes with a percentage of identity above $99 \%$ were post-processed as described in (39). Representatives for each family were selected by choosing the sequence with the fewest differences to the others on average and searched using tblastn (percentage of identity $>=80 \%$, genes from each reference were merged into pseudocontigs and grouped by gene composition.

203 For each family, a representative was selected as before and searched/filtered using the 204 references as before. The final core gene set contains 1,542 sequences that span a total of $2051,470,119$ nucleotides (approximately $67 \%$ of a typical $N$. gonorrhoeae genome length, 2.2Mb). 206 A BLAST database was constructed from these core segments and used to profile new 207 assemblies.

\section{Profiling new assemblies}

209 New genome assemblies can be uploaded by a user (drag and drop) or calculated from high210 throughput short read data directly within Pathogenwatch using SPAdes (40) as described in (41).

211 A taxonomy assignment step for species identification is performed on the uploaded assemblies

212 by using Speciator (42). New assemblies are then queried against a species-specific BLAST 213 database using blastn. For N. gonorrhoeae, every core loci needs to match at least $80 \%$ of its 214 length to be considered as present. Further filtering steps are applied to remove loci that can be 
215 problematic for tree building, such as paralogs or loci with unusually large number of variant sites

216 compared to an estimated substitution rate on the rest of the genome, as described in (43). The

217 overall substitution rate is calculated as the number of total differences in the core library divided

218 by the total number of nucleotides. Indels are ignored to minimise the noise that could be caused

219 by assembly or sequencing errors. The expected number of substitutions per locus is determined

220 by multiplying this substitution rate by the length of the representative sequence.

221 The number of substitutions observed for each locus between the new assembly and the reference sequence are scaled to the total number of nucleotides that match the core library, creating a pairwise score that is saved on a distance matrix and is used for Neighbour-Joining tree construction, as described in (44).

\section{Algorithms for sequence typing and cgMLST clustering}

226 Alleles and sequence types (STs) for Multi-Locus Sequence Typing (MLST) (45) and cgMLST

227 (core genome MLST, N. gonorrhoeae cgMLST v1.0) (46) were obtained from PubMLST $(47,48)$, 228 for N. gonorrhoeae Multi-Antigen Sequence Typing (NG-MAST) (49) from (50) and for $N$. 229 gonorrhoeae Sequence Typing for Antimicrobial Resistance (NG-STAR) (51) from (52) (Table 1). 230 A search tool implemented as part of Pathogenwatch is used to make the assignments for MLST, cgMLST and NG-STAR, while NGMASTER (53) is used for NG-MAST. Briefly, exact matches to known alleles are searched for, while novel sequences are assigned a unique identifier. The

233 combination of alleles is used to assign a ST as described in (54). Databases are regularly 234 updated and novel alleles and STs should be submitted by the user to the corresponding schemes 235 for designation.

236 cgMLST typing information is used for clustering individual genomes with others in the 237 Pathogenwatch database using single linkage clustering as described in (55). Users can select 238 the clustering threshold (i.e. number of loci with differing alleles) and a network graph based on 239 the SLINK (56) algorithm is calculated within individual genome reports. 


\section{AMR library and detection of genetic AMR determinants}

242 Genes and point mutations (single nucleotide polymorphisms (SNPs) and indels) were detected 243 using Pathogenwatch AMR v2.4.9 (57). Pathogenwatch AMR also provides a prediction of AMR phenotype inferred from the combination of identified mechanisms. Genetic determinants described in the literature as involved in AMR in N. gonorrhoeae were collated into a library in

246 TOML format (version 0.0.11). A test dataset containing 3,987 isolates from 13 studies $(15,18$, 22, 58-67) (Additional file 1: Table S1) providing minimum inhibitory concentration (MIC) information for six antibiotics (benzylpenicillin, tetracycline, ciprofloxacin, cefixime, ceftriaxone and azithromycin) was used to benchmark and to curate this library. A validation benchmark was posteriorly run with a dataset of 1,607 isolates from 3 other publications (68-70) with MIC information for the same six antibiotics plus spectinomycin (Additional file 1: Table S1). EUCAST clinical breakpoints v9.0 (71) were used to define susceptibility (S), susceptibility with an increased exposure $(\mathrm{I})$ or resistance $(\mathrm{R})(\mathrm{SIR})$ categorical interpretations of MICs for all antibiotics except for azithromycin, for which the EUCAST epidemiological cut-off (ECOFF) was used to define non-susceptibility/resistance (ECOFF $>1 \mathrm{mg} / \mathrm{L})$. As a result of the benchmark analyses, sensitivity, specificity and positive/negative predictive values (PPV/NPV) were obtained for the AMR mechanisms implemented in the library and, globally, for each of the antibiotics. Confidence intervals $(95 \%)$ for these statistics were calculated using the epi.tests function in the epiR $\mathrm{R}$ package v1.0-14 (72). Individual or combined AMR mechanisms with a PPV below 15\% were discarded from the library to optimise the overall predictive values. Visual representations of the observed ranges of MIC values for a particular antibiotic for each of the observed combinations of genetic AMR mechanisms on the test dataset were used to identify and assess combinations of mechanisms that have an additive or suppressive effect on AMR. These were included in the library.

265 As part of the accuracy testing of the AMR library, we ran the 2016 WHO N. gonorrhoeae 266 reference genomes 2016 panel $(n=14)$ through Pathogenwatch and compared the detected list 267 of genetic AMR mechanisms with the list published in the original study (22). For the WHO $U$ 
strain, a discrepancy on a mutation in parC was further investigated by mapping the original raw

269 Illumina data (European Nucleotide Archive (ENA) run accession ERR449479) to the reference

270 genome assembly (ENA genome accession LT592159.1) and visualized using Artemis (73).

271 In short-read assemblies, the four copies of the 23S rRNA gene are collapsed into one, thus the

272 detection of the A2045G and C2597T mutations is dependent on the consensus bases resulting

273 from the number of mutated copies $(63,66,74)$.

\section{Quality check and assembly of public sequencing data}

275 Public N. gonorrhoeae genomes with geolocation data were obtained from the ENA in November 2019. This list was complemented by an exhaustive literature search of studies on N. gonorrhoeae genomics without metadata submitted to the ENA but instead made available as supplementary information in the corresponding publications. Raw paired-end short read data from a list of 12,192 isolates was processed with the GHRU assembly pipeline v1.5.4 (75). This pipeline runs a Nextflow workflow to quality-check (QC) paired-end short read fastq files before and after filtering and trimming, assembles the data and quality-checks the resulting assembly. Results from the pipeline are provided in Additional file 2. In this pipeline, QC of short reads was performed using FastQC v0.11.8 (76). Trimming was done with Trimmomatic v0.38 (77) by cutting bases from the start and end of reads if they were below a Phred score of 25 , trimming using a sliding window of size 4 and cutting once the average quality within the window fell below a Phred score of 20 . Only reads with length above a third of the original minimum read length were kept for further analyses. After trimming, reads were corrected using the kmer-based approach implemented in Lighter v1.1.1 (78) with a kmer length of 32 bp and a maximum number of corrections allowed within a $20 \mathrm{bp}$ window of 1 . ConFindr v0.7.2 was used to assess intra- and inter-species contamination (79). Mash v2.1 (80) was applied to estimate genome size using a kmer size of $32 \mathrm{bp}$ and Seqtk v1.3 (81) to down sample fastq files if the depth of coverage was above 100x. Flash v1.2.11 (82) was used to merge reads with a minimum overlap length of $20 \mathrm{bp}$ and a maximum overlap of $100 \mathrm{bp}$ to facilitate the subsequent assembly process. SPAdes $\mathrm{v} 3.12$ 
294 (40) was used for genome assembly with the --careful option selected to reduce the number of

295 mismatches and short indels with a range of kmer lengths depending on the minimum read length.

296 The final assemblies were quality-checked using Quast v5.0.2 (83) and ran through the species

297 identification tool Bactinspector (84). QC conditions were assessed and summarised using

298 Qualifyr (85).

299 Fastq files with poor quality in which the trimming and filtering step discarded all reads from either

300 one or both pairs were excluded from the analyses because the assembly pipeline is optimised

301 for paired-end data. Assemblies with an N50 below $25,000 \mathrm{bp}$, a number of contigs above 300 , a

302 total assembly length above $2.5 \mathrm{Mb}$ or a percentage of contamination above $5 \%$ were also excluded.

\section{Metadata for public genomes}

305 Geolocation data (mainly country), collection dates (day, month and year when available), ENA 306 project accession and associated Pubmed ID were obtained from the ENA API for all the genomes 307 in the pipeline (86). A manual extensive literature search was performed to identify the 308 publications containing the selected genomes. In order to complete published studies as much 309 as possible, extra genomes were downloaded and added to the dataset. Metadata for the final set was completed with the information contained in supplementary tables on the corresponding

311 publications, including phenotypic antimicrobial susceptibility data. Submission date was

312 considered instead of collection date when the latter was not available, however, this occurred in 313 only a few cases $(<0.5 \%)$.

\section{Creation of the N. gonorrhoeae Pathogenwatch Scientific Steering Group}

315 International experts in the field of $N$. gonorrhoeae AMR, microbiology, genetics, genomics, 316 epidemiology and public health were approached and agreed to participate as members of the 317 'N. gonorrhoeae Pathogenwatch Scientific Steering Group' in order to discuss the analytics in 318 Pathogenwatch and make sure they met the current needs of the public health and scientific 319 community. During the updates made to the platform and the preparation of this manuscript, these 
experts participated in virtual sessions to discuss the list of genetic AMR determinants and their association with SIR categories (Table 2) based on experimental and/or computational evidence.

322 Some of the members of the group had previously been directly involved in many of these studies.

323 Other current and future updates were also discussed, such as the inclusion of the NG-STAR typing scheme (51) and the organization of published genomes into public collections, data sharing, privacy and the interconnectivity of Pathogenwatch with other platforms, such as PubMLST (48) or the ENA. The group will regularly discuss new updates to the platform.

\section{Data sharing and privacy}

328 Sequencing data and metadata files uploaded to Pathogenwatch by the user are kept within the user's private account. Genomes can be grouped into collections and these can be toggled between private and accessible to collaborators via a URL. Collection URLs include a 12-letter random string to secure them against brute force searching. Setting a collection to 'off-line mode' allows users to work in challenging network conditions, which may be beneficial in LMICs - all data are held within the browser. Users can also integrate private and potentially confidential metadata into the display without uploading it to the Pathogenwatch servers (locally within the browser on a user's machine).

\section{Results}

\section{N. gonorrhoeae genome analytics in Pathogenwatch}

339 Pathogenwatch is a web-based platform for epidemiological surveillance using genome sequencing data. After upload, different analytics are run simultaneously (Figure 1): cgMLST (46),

341 MLST (45), NG-MAST (49) and NG-STAR (51) typing schemes (Table 1), a genotypic prediction

342 of phenotypic resistance using a customized AMR library (Table 2) that includes known genetic 343 AMR mechanisms for 8 antimicrobials, as well as statistics on the quality of the assemblies 344 (Additional file 3: Figure S1). These analytical features differentiate Pathogenwatch from a parallel 
345 platform from the same group, Microreact (37), which shares one of the main layouts with

346 Pathogenwatch (a phylogenetic tree, a map and a table or timeline), but it is intended for

347 visualization of pre-computed phylogenetic trees with accompanying metadata, while

348 Pathogenwatch also includes analytical tools.

349 Genomes from one or multiple studies can be grouped into collections (Figure 2 and Additional

350 file 3: Figure S2), and the genomic data are automatically processed by comparing with a core $N$.

351 gonorrhoeae genome built from WHO reference strain genomes $(15,22)$. A phylogenetic tree is

352 obtained as a result, representing the genetic relationship among the isolates in the collection.

353 Metadata can be uploaded at the same time as the genome data, and if the collection location

354 coordinates for an isolate are provided, this information is plotted into a map (Additional file 3:

355 Figure S1). If date or year of isolation is also provided, this information is represented in a timeline.

356 The three panels on the main collection layout - the tree, the map and a table or timeline - are

357 functionally integrated so filters and selections made by the user update all of them

358 simultaneously. Users can also easily switch among the metadata and the results of the main

359 analytics: typing, genome assembly statistics, genotypic AMR prediction, AMR-associated SNPs,

360 AMR-associated genes and the timeline (Additional file 3: Figure S1). cgMLST is used for finding

361 close genomes in the database based on allele differences to one individual isolate (Additional

362 file 3: Figure S3). A video demonstrating the usage and main features of Pathogenwatch is

363 available (87). Notes on data sharing and privacy are available in the Methods section. 

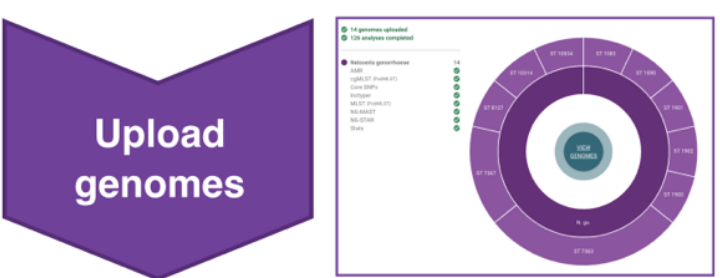

- Drag and drop new assemblies (fasta) or reads (fastq) to upload.

- After upload, typing and AMR modules are run automatically.
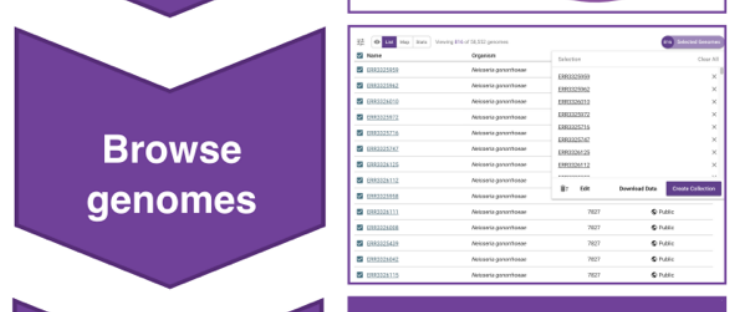

- List of public and private genomes with species identification, MLST, country and date information.

- Map and assembly statistics.

- Group genomes into collections.
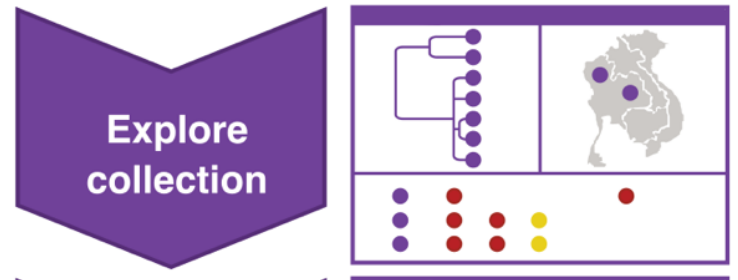

- Collection tree of selected genomes.

- Map and timeline.

- Metadata table.

- Typing: MLST, NG-STAR, NG-MAST.

- AMR: Antibiotics, Genes, SNPs.
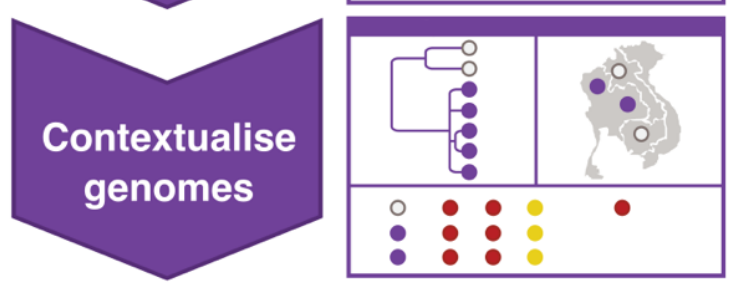

- Contextualise new genomes with other public or private genomes.
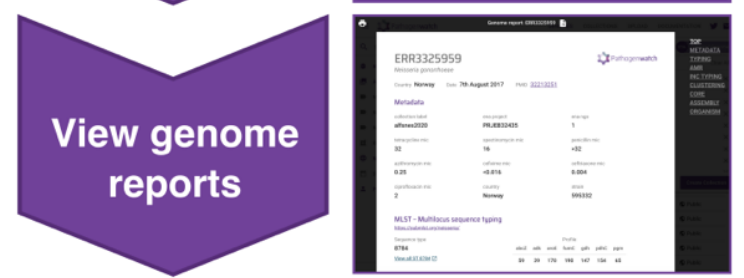
studies available.
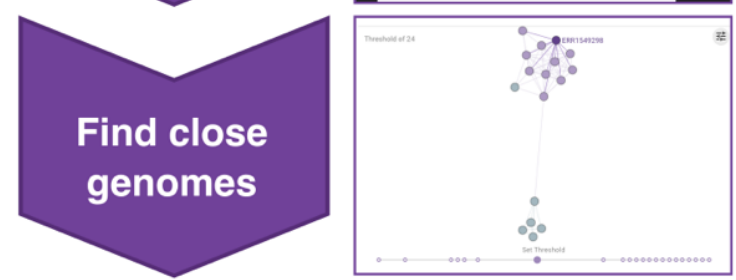

Individual genome reports containing:

- Species identification and metadata.

- Typing: MLST, NG-STAR, NG-MAST.

- AMR: genetic determinants/prediction.

- cgMLST-based clustering.

Figure 1. Main workflow in Pathogenwatch. New genomes can be uploaded and combined with public data for contextualisation. The collection view allows data exploration through a combined phylogenetic tree, a map, a timeline and the metadata table, which can be switched to show typing information (Multi-Locus Sequence Typing, MLST; $N$. gonorrhoeae Sequence Typing for Antimicrobial Resistance, NG-STAR; and N. gonorrhoeae Multi-Antigen Sequence Typing, NG-MAST) as well as known genetic AMR mechanisms for eight antibiotics. Genome reports summarise the metadata, typing and AMR marker results for individual isolates and allow finding other close genomes in 


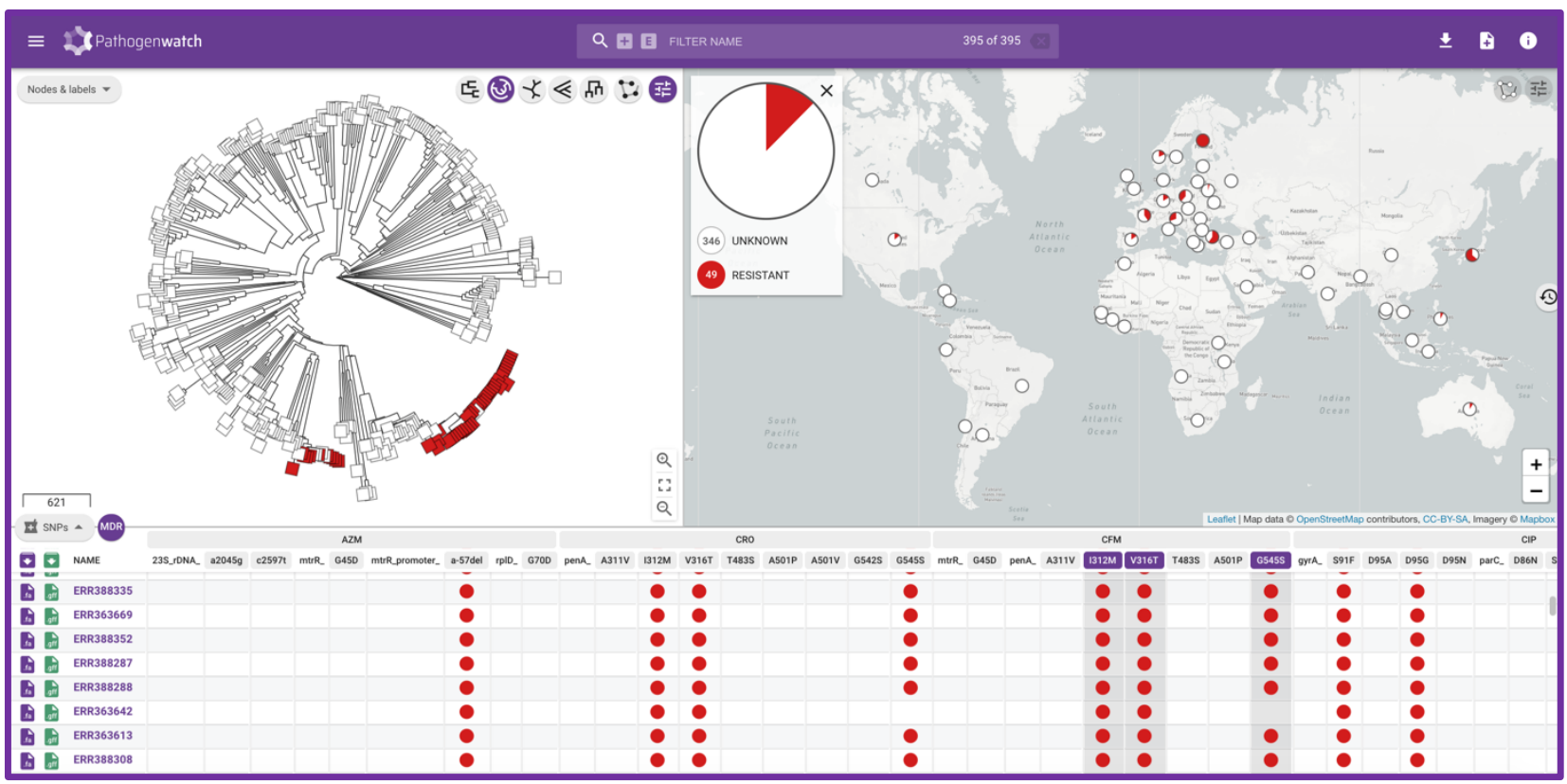

Figure 2. Main display of a Pathogenwatch collection, showing a phylogenetic tree, a map and a table of SNPs associated with AMR of $395 \mathrm{~N}$. gonorrhoeae genomes from a global study $(64,88)$. Isolates carrying three mosaic penA marker mutations are marked in red in the tree and the map. The table can be switched to show the metadata, a timeline, typing results (Multi-Locus Sequence Typing, MLST; N. gonorrhoeae Sequence Typing for Antimicrobial Resistance, NG-STAR and N. gonorrhoeae Multi-Antigen Sequence Typing, NG-MAST) as well as AMR analytics (known genetic mechanisms and genotypic AMR prediction) implemented in the platform. Further detail is shown in Additional file 3: Figure S1.

Table 1. N. gonorrhoeae sequence typing schemes implemented in Pathogenwatch.

\begin{tabular}{|c|c|c|c|c|}
\hline $\begin{array}{l}\text { Typing } \\
\text { scheme* }^{*}\end{array}$ & Loci (number) & Note & $\begin{array}{l}\text { Pathogenwatch } \\
\text { implementation }\end{array}$ & References \\
\hline cgMLST & $(\mathrm{N}=1,649)$ & $\begin{array}{l}\text { N. gonorrhoeae } \\
\text { cgMLST v1.0 }\end{array}$ & $\begin{array}{l}\text { Typing algorithm, database } \\
\text { from PubMLST }\end{array}$ & $(46-48,89)$ \\
\hline MLST & $\begin{array}{l}\text { abcZ, adk, aroE, } \\
\text { fumC, gdh, pdhC, } \\
\operatorname{pgm}(\mathrm{N}=7)\end{array}$ & $\begin{array}{l}\text { Housekeeping genes } \\
\text { in Neisseria spp. }\end{array}$ & $\begin{array}{l}\text { In-house typing tool, database } \\
\text { from PubMLST }\end{array}$ & $(45,47,48,89)$ \\
\hline NG-MAST & porB, tbpB $(\mathrm{N}=2)$ & $\begin{array}{l}\text { Genes encoding } \\
\text { highly-variable } \\
\text { membrane proteins }\end{array}$ & $\begin{array}{l}\text { NG-MASTER, database from } \\
\text { NG-MAST website }\end{array}$ & $(49,50,53)$ \\
\hline NG-STAR & $\begin{array}{l}\text { penA, mtrR, porB, } \\
\text { ponA, gyrA, parC, } \\
23 S \text { rDNA }(\mathrm{N}=7)\end{array}$ & $\begin{array}{l}\text { Genes involved in } \\
\text { antimicrobial } \\
\text { resistance }\end{array}$ & $\begin{array}{l}\text { In-house typing tool, database } \\
\text { from NG-STAR website }\end{array}$ & $(51,52,89)$ \\
\hline
\end{tabular}


Table 2. List of $N$. gonorrhoeae genetic antimicrobial resistance (AMR) determinants in Pathogenwatch. References that report evidence of association of each mechanism to AMR in clinical isolates and/or where their role on AMR has been confirmed in the laboratory through, e.g. transformation experiments, are included in the table. Effect: $R=$ resistance, $\mathrm{I}$ = susceptibility but increased exposure, $\mathrm{A}=$ additive effect, $\mathrm{N}=$ negative effect. $\mathrm{R}$ and I follow the EUCAST

\begin{tabular}{|c|c|c|c|}
\hline $\begin{array}{l}\text { Antibiotic } \\
\text { (MIC breakpoint } \\
\mathrm{mg} / \mathrm{L} \text { ) }\end{array}$ & Genetic AMR determinants & Effect & $\begin{array}{l}\text { Evidence } \\
\text { (References) }\end{array}$ \\
\hline Azithromycin & 23S rDNA 2045A>G substitution (2059A>G in E. coli) & $\mathrm{R}$ & $(74)$ \\
\hline \multirow[t]{17}{*}{$(\mathrm{R}: \mathrm{MIC}>1, \mathrm{ECOFF})$} & 23S rDNA $2597 \mathrm{C}>\mathrm{T}$ substitution (2611C>T in E. coli) & $\mathrm{R}$ & (90) \\
\hline & ermA, ermB, erm $C$, ermF genes & $\mathrm{R}$ & $(91,92)$ \\
\hline & ere $A$, ere $B$ genes & $\mathrm{R}$ & $(22)$ \\
\hline & mefA gene & $\mathrm{R}$ & $(92,93)$ \\
\hline & macAB promoter $-48 \mathrm{G}>\mathrm{T}$ substitution* & $\mathrm{R}$ & (94) \\
\hline & mtrR promoter mosaic** & & \\
\hline & N. meningitidis-like mosaic $(n=1)$ & $\mathrm{R}$ & $(23)$ \\
\hline & N. lactamica-like mosaic $(\mathrm{n}=2)$ & $\mathrm{R}$ & (23) \\
\hline & $m t r D$ mosaic $^{* *}$ & & \\
\hline & N. meningitidis-like mosaic $(n=1)$ & $\mathrm{R}$ & $(23)$ \\
\hline & N. lactamica-like mosaic $(\mathrm{n}=2)$ & $\mathrm{R}$ & (23) \\
\hline & $m t r R$ promoter -57 delA* & A & $(95,96)$ \\
\hline & $m t r R$ G45D & A & $(97,98)$ \\
\hline & $m t r C$ loss-of-function & $\mathrm{N}$ & $(26)$ \\
\hline & rp/V ARAK tandem duplication (position 90) & $\mathrm{R}$ & $(18)$ \\
\hline & rp/V KGPSLK tandem duplication (position 83) & $\mathrm{R}$ & (18) \\
\hline & $r p / D$ G70D & A & (25) \\
\hline \multirow{4}{*}{$\begin{array}{l}\text { Ceftriaxone } \\
(\mathrm{R}: \mathrm{MIC}>0.125)\end{array}$} & penA mosaic (A311V, I312M, V316P/T, T483S and G545S) & $\mathrm{R}$ & $(99-101)$ \\
\hline & penA V316P, T483S, A501P/V, G542S & $\mathrm{R}$ & $(99,100)$ \\
\hline & rрoB P157L, G158V, R201H & $\mathrm{R}$ & $(21)$ \\
\hline & rpoD D92-95 deletion, E98K & I & $(21)$ \\
\hline \multirow{6}{*}{$\begin{array}{l}\text { Cefixime }^{* * *} \\
(R: \text { MIC>0.125) }\end{array}$} & $m t r R$ G45D & A & $(97,98)$ \\
\hline & penA mosaic (I312M, V316T, G545S) & $\mathrm{R}$ & $(99-101)$ \\
\hline & penA mosaic (A311V, I312M, V316P/T, T483S and G545S) & $\mathrm{R}$ & $(99-101)$ \\
\hline & penA V316P, T483S, A501P & I & $(99,100)$ \\
\hline & rров P157L, G158V, R201H & I & (21) \\
\hline & rpoD D92-95 deletion, E98K & I & $(21)$ \\
\hline \multirow{6}{*}{$\begin{array}{l}\text { Ciprofloxacin } \\
(\mathrm{I}: 0.03<\mathrm{MIC} \leq 0.06 \text {; } \\
\mathrm{R}: \mathrm{MIC}>0.06)\end{array}$} & gyrA S91F, D95A/N & $\mathrm{R}$ & $(102)$ \\
\hline & gyrA D95G & I & $(102)$ \\
\hline & norM promoter $-7 \mathrm{~A}>\mathrm{G},-104 \mathrm{C}>\mathrm{T}$ substitutions ${ }^{*}$ & I & $(103)$ \\
\hline & parC D86N, S87R & $\mathrm{R}$ & $(102)$ \\
\hline & parC S87I/N, S88P, E91K & I & $(102)$ \\
\hline & parE G410V & I & $(104)$ \\
\hline \multirow{6}{*}{$\begin{array}{l}\text { Tetracycline } \\
(\mathrm{I}: 0.5<\mathrm{MIC} \leq 1 \\
\mathrm{R}: \mathrm{MIC}>1)\end{array}$} & $m t r R$ A39T, G45D & A & $(97,98)$ \\
\hline & $m t r R$ loss-of-function & I & $(22)$ \\
\hline & $m t r R$ promoter $-56 \mathrm{~A}>\mathrm{C}$ substitution, -57 delA deletion* & I & $(23,95,96)$ \\
\hline & $m t r R$ promoter $-131 \mathrm{G}>\mathrm{A}(m t r C-120 \mathrm{G}>\mathrm{A}$ substitution, $m t r 120) *$ & I & $(97)$ \\
\hline & rpsJ V57M & I & $(105)$ \\
\hline & tetM gene & $\mathrm{R}$ & $(106)$ \\
\hline
\end{tabular}




\begin{tabular}{|c|c|c|c|}
\hline Penicillins & blaTEM gene & $\mathrm{R}$ & $(107)$ \\
\hline (I: $0.06<\mathrm{MIC} \leq 1$ & $m t r R$ G45D & I & $(97,98)$ \\
\hline \multirow[t]{8}{*}{$\mathrm{R}: \mathrm{MIC}>1)$} & $m t r R$ A39T & A & $(97)$ \\
\hline & $m t r R$ loss-of-function & I & (22) \\
\hline & $m t r R$ promoter $-56 \mathrm{~A}>\mathrm{C},-57 \mathrm{del} \mathrm{A}^{*}$ & i & $(23,96)$ \\
\hline & $m t r R$ promoter $-131 \mathrm{G}>\mathrm{A}(m t r C-120 \mathrm{G}>\mathrm{A} \text { substitution, } m t r 120)^{*}$ & I & (97) \\
\hline & $\begin{array}{l}\text { penA I312M, V316P/T, ins346D, T483S, A501P/T/V, G542S, } \\
\text { G545S, P551S }\end{array}$ & I & $(99,100)$ \\
\hline & penA mosaic (I312M, V316T, G545S) & A & $(99-101)$ \\
\hline & ponA1 L421P & 1 & $(108)$ \\
\hline & porB1b G120K, A121N/D & I & $(109)$ \\
\hline \multirow{3}{*}{$\begin{array}{l}\text { Spectinomycin } \\
(\mathrm{R}: \mathrm{MIC}>64)\end{array}$} & $16 S$ rDNA $1184 C>T(1192 C>T$ in E. coli $)$ & $\mathrm{R}$ & $(110)$ \\
\hline & $r p s E \mathrm{~T} 24 \mathrm{P}$ & $\mathrm{R}$ & $(111)$ \\
\hline & rpsE V27- deletion, K28E & $\mathrm{R} / \mathrm{A}$ & (111) \\
\hline$\underset{* \star * * * *}{\text { Sulfonamides }}$ & folP R228S & $\mathrm{R}$ & $(22,112)$ \\
\hline
\end{tabular}

*Nomenclature of the mutations on the $\operatorname{mac} A B, \operatorname{mtrR}$ and norM promoter regions is based on $N$. gonorrhoeae coordinates considering the distance from the start of the macAB, mtrR and norM genes, respectively. ${ }^{*}$ Note that mosaics are caused by recombination events, which can have variable breakpoints with different effects on azithromycin MIC if any. In this version, we have included the three mosaics described by Wadsworth et al. (23), but the list will be expanded as new mosaic mtr (intergenic region between $m t r R$ and $m t r C$ ) and $m t r D$ alleles having an effect on azithromycin MICs are published. ${ }^{* * *}$ The list of genetic AMR mechanisms for the ESCs ceftriaxone and cefixime do not include all known porB1b or $m t r R$-associated variants as their effect was found not to be relevant in increasing MIC on the benchmark analyses for phenotypic AMR prediction purposes despite the experimental evidence reported in Zhao et al. (113). In case of strains carrying penA-associated mutations, their immediate predicted phenotype is that of those carrying penA-associated variants. ${ }^{* * *}$ The list of genetic AMR mechanisms for tetracycline does not include porB1b mutations as their effect

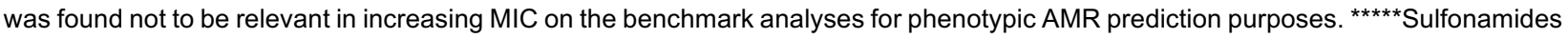
are not a treatment alternative for gonorrhoea, however the folP R228S mutation is kept in this version of the library for surveillance purposes.

\section{Library of genetic AMR mechanisms: genotypic and phenotypic benchmarks}

423 We compiled described genetic AMR mechanisms previously reported for $N$. gonorrhoeae up to

424 the writing of this manuscript into the AMR library in Pathogenwatch (Table 2).A genotypic accuracy testing of the AMR library was performed using the $14 \mathrm{~N}$. gonorrhoeae reference genomes from the WHO 2016 panel (22), which were uploaded into Pathogenwatch. All the genetic AMR determinants described as present in these isolates and implemented in the

428 Pathogenwatch AMR library were obtained as a result (Additional file 1: Table S2). Only one 429 discrepancy was found when compared to the original publication. The WHO $U$ strain was 430 reported as carrying a parC S87W mutation. However, mapping the original Illumina data from 431 this isolate with the final genome assembly revealed that this strain carries a wild type allele 432 (Additional file 3: Figure S4). MLST and NG-MAST types were the same as those reported in the 
identity to $N$. meningitidis and $89 \%$ to $N$. gonorrhoeae, and it is included as screening because it has previously been shown to cause false negative results in some molecular detection tests for N. gonorrhoeae (114).

Then, we also performed a genotypic-phenotypic benchmark using a test dataset of 3,987 $\mathrm{N}$.

439 gonorrhoeae isolates from 13 different studies containing MIC information for at least part of the 440 following six antibiotics: ceftriaxone, cefixime, azithromycin, ciprofloxacin, benzylpenicillin and 441 tetracycline (Additional file 1: Table S1). EUCAST clinical breakpoints were applied for five of the 442 antimicrobials except for azithromycin, for which the adoption of an ECOFF $>1 \mathrm{mg} / \mathrm{L}$ is now 443 recommended to distinguish isolates with azithromycin resistance determinants, instead of a 444 clinical resistance breakpoint $(115,116)$. A visualization of the range of MICs on each particular combination of genetic AMR mechanisms observed on the isolates from the benchmark test dataset (Figure 3a-b and Additional file 3: Figures S5-S10) revealed combinations that show an

447 additive effect on AMR. These combinations were included in the AMR library to improve the 448 accuracy of the genotypic prediction. For example, rpsJ V57M and some mtrR-associated 449 mutations individually are associated with a decreased susceptibility or intermediate resistance 450 to tetracycline (MICs of $0.5-1 \mathrm{mg} / \mathrm{L}$ ), however, a combination of these variants can increase MICs above the EUCAST resistance breakpoint for tetracycline (MICs>1 mg/L) (Additional file 3: Figure

452 S9). This is the case of the combination of $r p s J \mathrm{~V} 57 \mathrm{M}$ with the $m t r R$ promoter -57 delA mutation 453 ( $\mathrm{N}=681$ isolates, $94.9 \%$ positive predictive value, $\mathrm{PPV}$ ) or with $m t r R$ promoter -57 delA and $m t r R$ 454 G45D ( $\mathrm{N}=83$ isolates, 93.9\% PPV). Several combinations of penA, ponA1, mtrR and porB1b 455 mutations were observed to be able to increase the benzylpenicillin MIC above the resistant 456 threshold in most of the cases (Additional file 3: Figure S10). This is the case of the porB1b 457 mutations combined with $m t r R$ A39T ( $N=31$ isolates, $100 \%$ PPV), with the $m t r R$ promoter -57 delA deletion ( $\mathrm{N}=286$ isolates, 96.5\% PPV) or with $m$ trR promoter -57 delA and ponA1 L421P ( $\mathrm{N}=269$ 459 isolates, $96.3 \%$ ). Despite mosaic penA not being a main driver of resistance to penicillins, a 460 combination of the porB1b mutations with the three main mosaic penA mutations (G545S, I312M and V316T) was also associated with a resistant phenotype in all cases $(\mathrm{N}=17$ isolates, $100 \%$ 
bioRxiv preprint doi: https://doi.org/10.1101/2020.07.03.186726; this version posted December 4, 2020. The copyright holder for this preprint (which was not certified by peer review) is the author/funder, who has granted bioRxiv a license to display the preprint in perpetuity. It is made available under aCC-BY-NC-ND 4.0 International license.

462

463

464

465

466

467

PPV). A recent publication showed that loss-of-function mutations in $m t r C$ increased susceptibility to azithromycin and are associated with isolates from the cervical environment (26). We included the presence of a disrupted $m t r C$ as a modifier of antimicrobial susceptibility in the presence of an mtr mosaic, as we did not have enough evidence from the test dataset to assess the MIC ranges of isolates with the 23S rDNA A2045G and C2597T mutations with and without a disrupted mtrC gene.

a

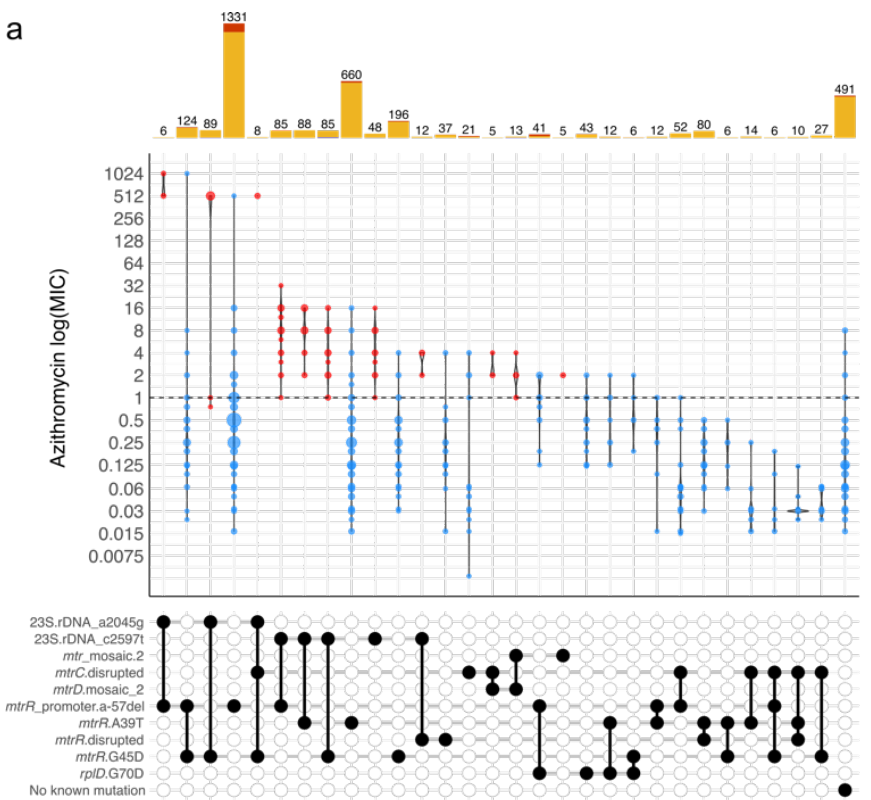

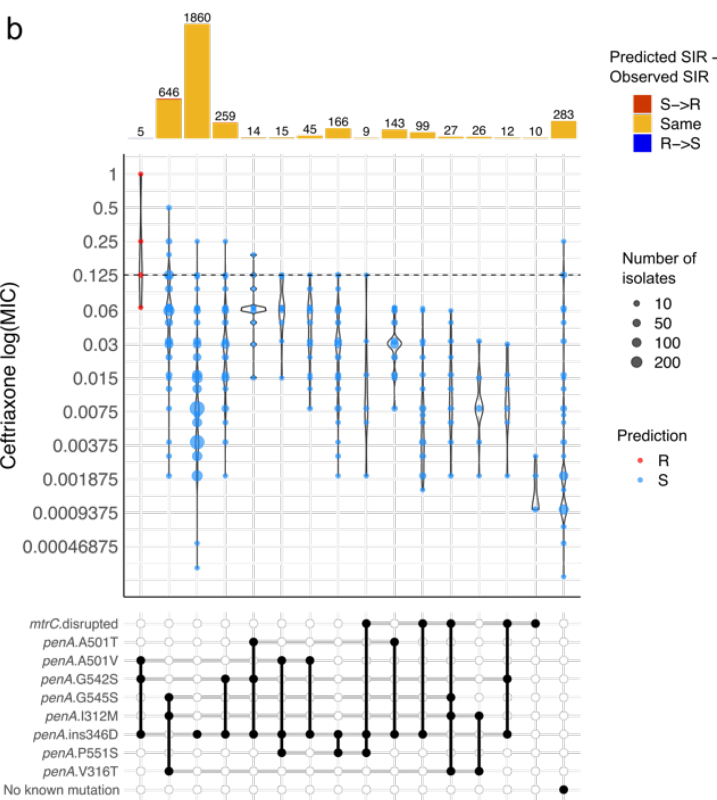

C
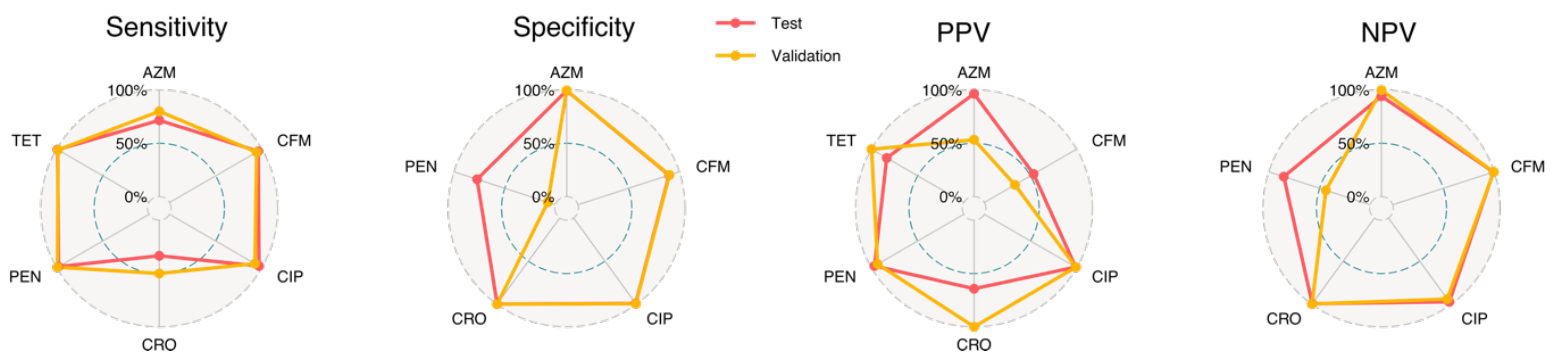

Figure 3. Distribution of minimum inhibitory concentration (MIC) values $(\mathrm{mg} / \mathrm{L})$ for the last-line antibiotics for $N$. gonorrhoeae azithromycin (a) and ceftriaxone (b) in a collection of 3,987 N. gonorrhoeae isolates with different combinations of genetic antimicrobial resistance (AMR) mechanisms. Only combinations observed in at least 5 isolates are shown (see Additional file 3: Figure S5-S10 for expanded plots for six antibiotics). Dashed horizontal lines on the violin plots mark the EUCAST epidemiological cut-off (ECOFF) for azithromycin and EUCAST clinical breakpoint for ceftriaxone. Point colours inside violins represent the genotypic AMR prediction by Pathogenwatch on each combination of mechanisms (indicated by black circles connected vertically; horizontal thick grey lines connect combinations of mechanisms that share an individual determinant). Barplots on the top show the abundance of isolates with each combination of mechanisms. Bar colours represent the differences between the predicted and the observed SIR (i.e. red for a predicted susceptible mechanism when the observed phenotype is resistant). (c) Radar plots 
and validation benchmark analyses. $\mathrm{AZM}=$ Azithromycin, $\mathrm{CFM}=$ Cefixime, $\mathrm{CIP}=$ Ciprofloxacin, $\mathrm{CRO}=$ Ceftriaxone, PEN = Benzylpenicillin, TET $=$ Tetracycline .

484 Results from the benchmark (Additional file 1: Table S3) show sensitivity values (true positive rates, $\mathrm{TP} /(\mathrm{TP}+\mathrm{FN})$; TP=True Positives, FN=False Negatives) above $96 \%$ for tetracycline $(99.2 \%)$, benzylpenicillin (98.1\%), ciprofloxacin $(97.1 \%)$ and cefixime $(96.1 \%)$, followed by azithromycin $(71.6 \%)$ and ceftriaxone $(33.3 \%)$. These results reflect the complexity of the resistance mechanisms for azithromycin and ceftriaxone, where the known genetic determinants explain only part of the antimicrobial susceptibility. However, specificity values (true negative rates, $\mathrm{TN} /(\mathrm{TN}+\mathrm{FP})$; $\mathrm{TN}=$ True Negatives, FP=False Positives) for these two antibiotics as well as ciprofloxacin were above 99\% (Additional file 1: Table S3), demonstrating that the genetic mechanisms included in the database have a role in AMR. The specificity value for cefixime was lower but nearly $90 \%$, mainly due to the high number of isolates with an MIC below the threshold but with three mutations characterising a mosaic penA allele (G545S, I312M and V316T, TP=367, $\mathrm{TN}=323, \mathrm{PPV}=53.2 \%$; Additional file 1: Table S4). Benzylpenicillin and tetracycline showed specificity values of $77.3 \%$ and $61.3 \%$, respectively. In the first case, all the mechanisms included in the library showed a PPV value above $94 \%$. For tetracycline, a considerable number of false positive results are mainly caused by the presence of $r p s J \mathrm{~V} 57 \mathrm{M}$, for which $\mathrm{PPV}=83.8 \%$ ( $T P=1083, \mathrm{FP}=209$; Additional file 1: Table S4). However, this mutation was kept in the AMR library because it can cause intermediate resistance to tetracycline on its own (Additional file 3:

501 Figure S9).

502 Results from the benchmark analysis on the 3,987-isolates dataset were used to curate and 503 optimize the AMR library. Thus, in order to objectively validate it, the benchmark analysis was 504 also run on a combination of three different collections $(\mathrm{N}=1,607$, Additional file 1: Table S1) with 505 available MIC information for seven antibiotics including spectinomycin (Additional file 1: Table 506 S3) $(69,70,117)$. Results from the test and validation benchmark runs were compared, showing that sensitivity values on the six overlapping antibiotics were very similar, with the validation 508 benchmark performing even better for azithromycin and ceftriaxone (Figure 3c). In terms of 
specificity, both datasets performed equally well for all antibiotics except for benzylpenicillin, in

510 which specificity drops in the validation benchmark. This is due to the penA_ins346D mutation

511 ( $T P=1125, F P=83)$ and the blaTEM genes $(T P=525, F P=36)$, which despite showing false

512 positives, have a PPV above 93\% (Additional file 1: Table S5). In general, discrepancies found

513 between the test and the validation benchmarks can be explained by particular mechanisms that

514 on their own show high predictive values and affect antibiotics for which we do not currently

515 understand all the factors involved in resistance, such as azithromycin and the ESCs (Additional

516 file 1: Table S5).

\section{Over 12,000 public genomes available}

518 Data for 11,461 isolates were successfully assembled and passed all quality cut-offs, resulting in

51912,515 isolates after including the previously-available Euro-GASP 2013 dataset (15). New 520 assemblies were uploaded and made public on Pathogenwatch, which now constitutes the largest repository of curated $N$. gonorrhoeae genomic data with associated metadata, typing and AMR information at the time of submission of this manuscript. Updated data spans 27 different publications $(18,53,58-61,63-65,67-70,117-131)$ and is organized into individual collections associated with the different studies (Additional file 1: Table S6). Available metadata was added

525 for the genomes from these publications while basic metadata fields were kept for others (country, year/date and ENA project number).

527 We cross-checked that the main clusters found in the phylogenetic trees obtained after creating

528 the public collections in Pathogenwatch were consistent with those observed in the trees in the 529 corresponding publications. For example, recent works defined two major clusters of $N$. 530 gonorrhoeae, termed Lineages A and B, which were found to be consistent with the corresponding 531 Pathogenwatch trees as exemplified for isolates from England in Town et al (2020) (68) (Figure

532 S11a). We were also able to differentiate the cefixime-resistant penA10 and penA34-carrying 533 clones from Vietnam from Lan et al (2020) (124) (Figure S11b) as well as the 10 major clusters 534 defined in the $N$. gonorrhoeae population circulating in New York City (NYC) as described in 
535 Mortimer et al (2020) (120) (Figure S11c). In the last case, we also liked to emphasize the

536 usefulness of Microreact (37) as a parallel tool to Pathogenwatch for more complex visualization

537 purposes, such as showing the 10 major clusters in NYC as metadata blocks of different colours.

538 The $N$. gonorrhoeae public data available on Pathogenwatch spans nearly a century (1928-2018)

539 and almost 70 different countries (Additional file 3: Figure S12). However, sequencing efforts are

540 unevenly distributed around the world, and over $90 \%$ of the published isolates were isolated in

541 only 10 countries, headed by the United Kingdom $(N=3,476)$, the United States $(N=2,774)$ and

542 Australia ( $\mathrm{N}=2,388$ ) (Additional file 1: Table S7, Figure 4). A total of 554 MLST, 1,670 NG-MAST

543 and 1,769 NG-STAR different STs were found in the whole dataset, from which a considerable

544 number were new profiles caused by previously undetected alleles or new combinations of known

545 alleles ( $N=92$ new MLST STs, $N=769$ new NG-STAR STs and $N=2,289$ isolates with new NG-

546 MAST porB and/or tbpB alleles). These new alleles and profiles were submitted to the

547 corresponding scheme servers. 


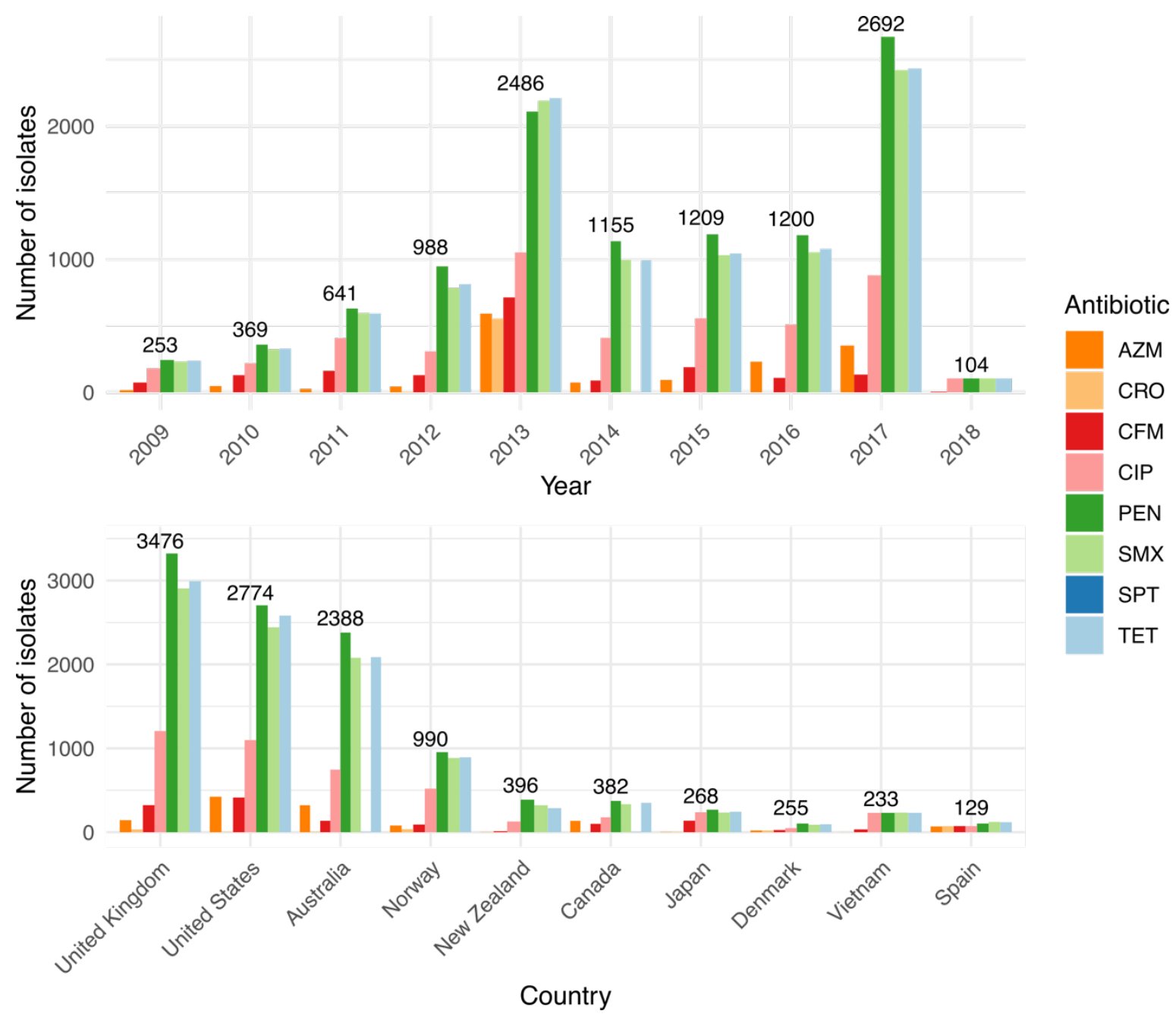

Figure 4. Summary of the geolocalization and collection date of 12,515 public $N$. gonorrhoeae genomes in Pathogenwatch. Coloured bars represent the genotypic antimicrobial resistance (AMR) prediction based on the mechanisms included in the library. $\mathrm{AZM}=$ Azithromycin, $\mathrm{CFM}=$ Cefixime, $\mathrm{CIP}=$ Ciprofloxacin, $\mathrm{CRO}=$ Ceftriaxone, $\mathrm{PEN}=$ Benzylpenicillin, TET $=$ Tetracycline .

549 Genomic studies are often biased towards AMR isolates, and this is reflected in the most abundant STs found for the three typing schemes within the public data. Isolates with MLST

551 ST1901, ST9363 and ST7363, which contain resistance mechanisms to almost every antibiotic

552 included in the study, represent over $25 \%$ of the data (Figure 5). Isolates with MLST ST1901 and

553 ST7363 are almost always associated with resistance to tetracycline, sulfonamides, benzylpenicillin and ciprofloxacin and nearly $50 \%$ of isolates from these two types harbour resistance mechanisms to cefixime. Ciprofloxacin resistance is not widespread among ST9363 isolates, which are associated with azithromycin resistance in nearly $50 \%$ of the isolates for this 
557 ST (Figure 5). NG-STAR ST63 (carrying the non-mosaic penA-2 allele, penA A517G and mtrR

558 A39T mutations as described in (52)) is the most represented in the dataset and carries resistance

559 mechanisms to tetracycline, sulfonamides, and benzylpenicillin, but is largely susceptible to

560 spectinomycin, ciprofloxacin, the ESCs cefixime and ceftriaxone and azithromycin. NG-STAR

561 ST90 isolates, conversely, are largely associated with resistance to cefixime, ciprofloxacin and

562 benzylpenicillin as they carry the key resistance mutations in mosaic penA-34, as well as in the

563 mtrR promoter, porB1b, ponA, gyrA and parC (as described in (52)). NG-MAST ST1407 is

commonly associated with MLST ST1901 and is the second most represented ST in the dataset

565 following NG-MAST ST2992, which mainly harbours resistance to tetracycline, benzylpenicillin

566 and sulfonamides (Figure 5).

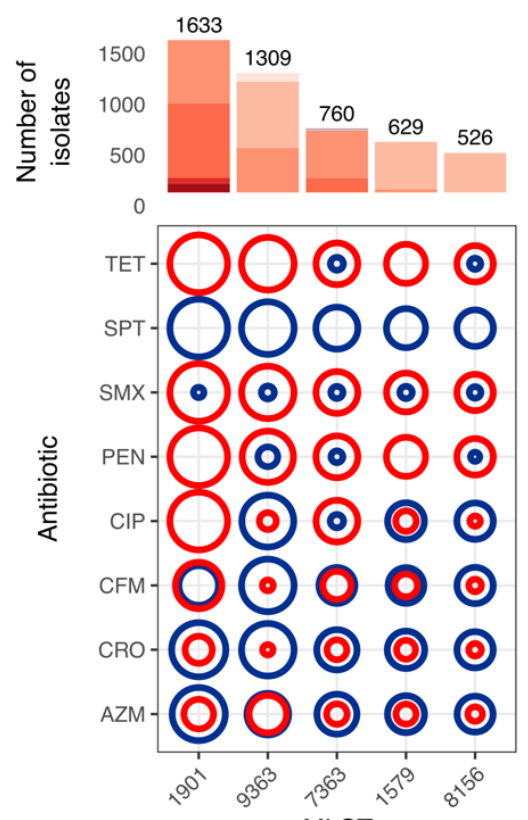

MLST
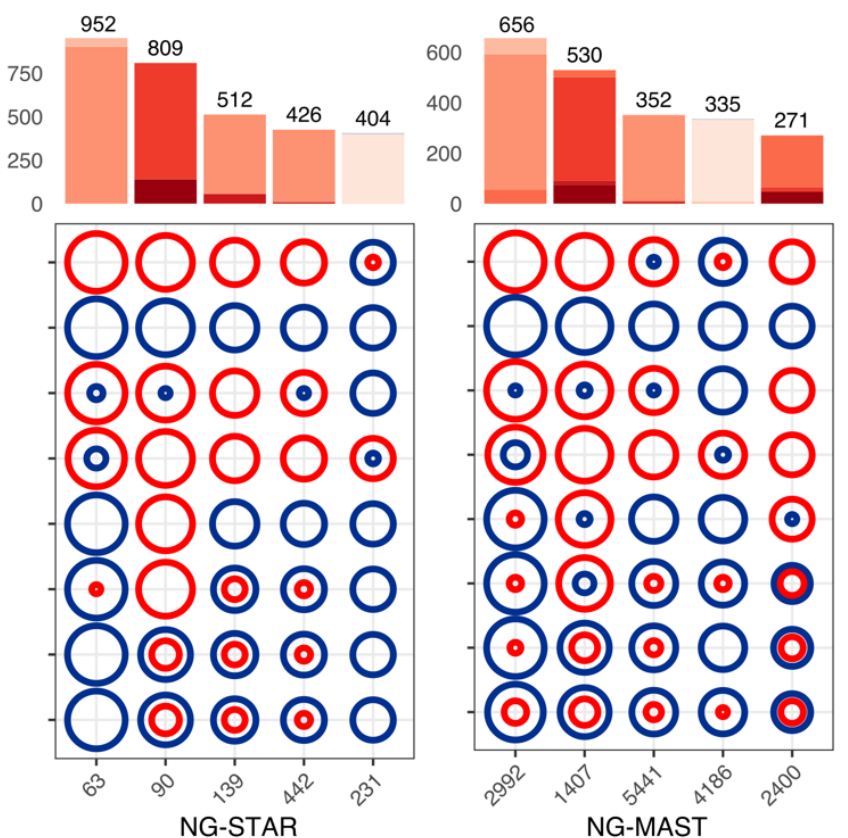

NG-MAST

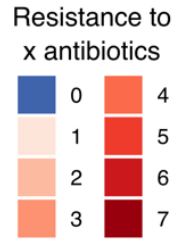

Frequency

- 10

(C) 100

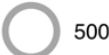

Antimicrobial susceptiblity

- $\mathrm{S}$

- R/l

Figure 5. Predicted antimicrobial resistance (AMR) profiles of the top five Multi-Locus Sequence Typing (MLST), N. gonorrhoeae Sequence Typing for Antimicrobial Resistance (NG-STAR) and N. gonorrhoeae Multi-Antigen Sequence Typing (NG-MAST) types in the N. gonorrhoeae public data in Pathogenwatch. Coloured circles in the grids show the proportion of genomes from each ST which are predicted to have an intermediate (susceptible but increased exposure) or resistant phenotype, red) versus susceptible genomes (in dark blue) from each sequence type (ST) and antibiotic. Bars on the top show the number of isolates from each ST coloured by the number of antibiotics the genomes are predicted to be resistant to. 


\section{Case study: global expansion of an mtr mosaic-carrying clone}

568 The genetic mechanisms that have commonly been associated with an increased MIC of 569 azithromycin in N. gonorrhoeae are two mutations in the 23S rRNA gene (A2045G and C2597T 570 substitutions, in $N$. gonorrhoeae nomenclature) as well as mutations in $m t r R$ and its promoter 571 (132, 133). As described above, other mechanisms have also been recently discovered that 572 increase the MIC of azithromycin (Table 2), such as mosaicism affecting the efflux pump-encoding $573 m t r C D E$ genes and its repressor $m t r R$, mainly when the mosaic spans the $m t r R$ promoter region 574 and $m t r D$ gene $(23,24)$. Some studies have recently reported the local expansion of azithromycin575 resistant $N$. gonorrhoeae lineages carrying an $m t r$ mosaic in the USA $(122,123,134)$ and 576 Australia (118). However, the extent of the dispersion of this mechanism to other parts of the

577 world has not been studied yet. Here, using the public genomes of $N$. gonorrhoeae in 578 Pathogenwatch, we have been able to explore this question.

579 A total of 1,142 strains with genetic determinants of azithromycin resistance were selected in 580 Pathogenwatch and combined with 395 genomes from a global collection (64) for background 581 contextualization (see Pathogenwatch project in (135)) (Figure 6a). 571 of the strains predicted

582 to be resistant to azithromycin had some form of mosaic in the $m t r R$ promoter and/or $m t r D$ gene 583 of one of the three types described in Wadsworth et al. (2018) (23) and included in the 584 Pathogenwatch AMR library (Table 2). These mosaics have been experimentally proven to increase MIC of azithromycin above $1 \mathrm{mg} / \mathrm{L}$, which is the EUCAST ECOFF value as well as the

586 Clinical Laboratory and Standards Institute (CLSI) non-susceptibility breakpoint $(23,24)$. One of

587 the $N$. lactamica-like mosaics, termed here 'mtr_mosaic.2', was by far the most extended, as it 588 was found in 545 genomes spanning the $m t r R$ promoter and/or the $m t r D$ gene, with $521(95.6 \%)$ 589 of them spanning both regions. Twenty-five genomes contained a $N$. meningitidis-like mosaic $590 m t r R$ promoter and/or mtrD gene ('mtr_mosaic.1') and in only $9(36 \%)$ of them the mosaic 591 spanned both loci. The N. lactamica-like 'mtr mosaic.3' was only found in isolate ERR855360 592 (GCGS834) from Los Angeles (USA, 2012), which is where the reference sequence for this 
mosaic was extracted from. Of the studies where these $m t r$ mosaic-carrying genomes were

594 obtained from, only those from the USA and Australia specifically targeted and found this genetic

595 determinant of resistance. The rest did not target this mosaic and some of them found strains with

596 unexplained increased MICs of azithromycin $(69,121,129)$, which could partly be explained by

597 the presence of these $m t r$ mosaics.

598 We observed one main lineage carrying mosaic 2 in $m t r R$ promoter and $m t r D$ gene (Figure 6a)

599 with 520 genomes. Of those, only 3 and 8 isolates carried the 23S rDNA A2045G and C2597T

600 mutations, respectively. Interestingly, the first strain in the database with this type of mosaic dates

601 from 2006 (18), however, it was not until the end of 2011-2012 when this lineage started to expand

602 (Figure 6b). Despite the genomic data contained in Pathogenwatch being biased to the amount

603

of data sequenced and published from each country and year, we can easily infer that this lineage

604 has spread across the world as we detect cases in Australia $(n=293)(118)$, the USA $(n=195)(18$,

$605120,122,123)$, Norway $(n=19)(121)$, the United Kingdom $(n=11)(68,119)$, and Ireland $(n=3)$

606 (129). A strong association was found to the country of isolation (Figure 6c), with a broad diversity

607 of sublineages having spread across the USA (strains mostly isolated between 2012 and 2016).

608 In contrast, an expansion of a particular clone, likely from a single main introduction, was observed

609 to have occurred in Australia (strains isolated in 2017), followed by a further divergence of a

610 subclone within the country which correlates with the loss of the porB1b G120K and A121N

611 mutations (Figure 6d), likely through a recombination event. Despite epidemiological data not

612 being available for the Australian study (118), from their work we know that the clusters carrying

613 an $m$ tr mosaic were mostly linked to transmission between men, although bridging among MSM

614 and heterosexual populations was also observed.

615 The results from our case study show that there is an emerging lineage of $N$. gonorrhoeae that

616 has spread across the world and that is carrying a mosaic $m t r$ that has been associated with low-

617 to-medium resistance to azithromycin. This global lineage, as well as others that may emerge

618 carrying this or other genetic AMR mechanisms, has to be closely monitored. For this purpose,

619 an up-to-date genomic epidemiology tool such as Pathogenwatch, which includes a list of genetic 
620 AMR mechanisms approved by an expert group is a great resource for the scientific community.

621 At the moment, Pathogenwatch includes references for three types of mosaics in the mtrR

622 promoter and $m t r D$ genes that have been experimentally proven to increase MIC of azithromycin

$623(23,24)$, and the detection of these mosaics on new genomes respond to a set of similarity rules

624 (see Data availability section). However, we will keep the database updated with new 625 experimentally-confirmed reference sequences that may arise from further studies as it is still 626 unclear whether all mosaics affecting the mtrCDE efflux pump will cause a decreased susceptibility to azithromycin.

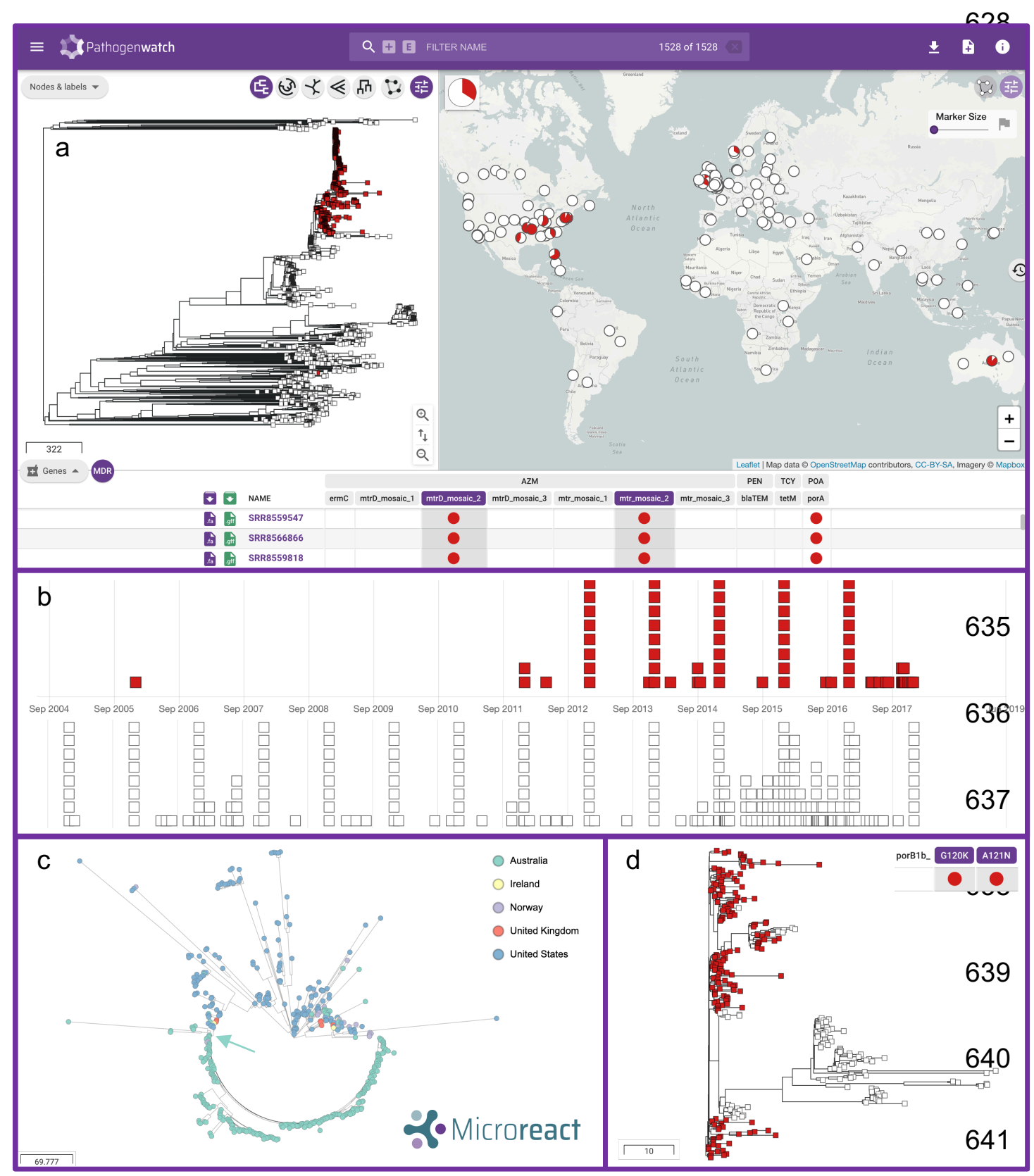


642 Figure 6. N. gonorrhoeae genomes carrying genetic AMR mechanisms associated to azithromycin resistance were

643 selected in Pathogenwatch $(n=1,142)$ and combined with genomes from a global collection $(64,88)($ total $n=1528)$ for

644 background contextualization. (a) Main layout of the combined collection, with the emerging lineage carrying $m t r$ mosaic

6452 spanning the $m t r R$ promoter and $m t r D$ marked in red in the tree and the map. (b) Timeline of the genomes carrying

646 mtr mosaic 2 (in red) and other public genomes in the database without this genetic AMR mechanism. (c) Visualization

647 of the $m$ tr mosaic 2-carrying lineage $(n=520)$ spreading in the USA and Australia (see legend) using Microreact. The 648 arrow in turquoise colour marks the divergence of the Australian lineage, shown in more detail in (d) coloured by the 649 presence (in red) or absence (in white) of the porB $1 b$ G120K and A121N mutations. The Pathogenwatch project of this 650 case study can be explored in (135).

\section{Discussion}

We present a public health focussed $N$. gonorrhoeae framework at Pathogenwatch, an open access platform for genomic surveillance supported by an expert group that can be adapted to any public health or microbiology laboratory. Little bioinformatics expertise is required, and users can choose to either upload raw short read data or assembled genomes. In both cases, the upload of high-quality data is encouraged in the form of quality-checked reads and/or quality-checked assemblies. Recent benchmark analyses show particular recommendations for long-read or hybrid data (136) as well as short read-only data $(40,137)$. On upload, several analyses are run on the genomes, and results for the three main typing schemes (MLST, NG-MAST and NG-STAR) as well as the detection of genetic determinants of AMR and a prediction of phenotypic resistance using these mechanisms can be obtained simultaneously. The library of AMR determinants contained in Pathogenwatch for $N$. gonorrhoeae has been revised and extended to include the latest mechanisms and epistatic interactions with experimental evidence of decreasing susceptibility or increasing resistance to at least one of eight antibiotics (Tables 2). A test and validation benchmark analyses revealed sensitivity and/or specificity values $>90 \%$ for most of the tested antibiotics (Additional file 1: Table S3). Sensitivity values for the antimicrobials in the current dual treatment, azithromycin (80\%) and ceftriaxone (50\%), reflect the complexity of the resistance mechanisms for these antibiotics, for which we can only explain part of the observed 670 phenotypic resistance. However, their specificity values were above 99\% (Additional file 1: Table 671 S3), further strengthening the associations of the included AMR determinants in increasing MICs 
672 of these antibiotics. It remains essential to perform phenotypic susceptibility testing so we can

673 detect inconsistencies between phenotypic and genotypic data that can lead to the identification

674 and subsequent verification of novel or unknown resistance mechanisms. This will allow to

675 continuously expand the list of genetic AMR mechanisms, and the AMR prediction from genomic

676 data will further improve.

677 The continuous increase in reporting of $N$. gonorrhoeae AMR isolates worldwide led to a call for 678 international collaborative action in 2017 to join efforts towards a global surveillance scheme. This 679 was part of the WHO global health sector strategy on STIs (2016-2021), which set the goal of 680 ending STI epidemics as a public health concern by year $2030(7,8)$. Several programmes are currently in place at different global, regional or national levels to monitor gonococcal AMR trends, emerging resistances and refine treatment guidelines and public health policies. This is the case

683 of, for example, the WHO Global Gonococcal Antimicrobial Surveillance Programme (WHO $684 \operatorname{GASP})(7,8)$, the Euro-GASP in Europe $(6,15,138)$, the Gonococcal Isolate Surveillance Project 685 (GISP) in the United States (139), the Canadian Gonococcal Antimicrobial Surveillance 686 Programme (140), the Gonococcal Surveillance Programme (AGSP) in Australia (141) or the 687 Gonococcal Resistance to Antimicrobials Surveillance Programme (GRASP) in England and 688 Wales (142). The WHO in collaboration with CDC has recently started an enhanced GASP 689 (EGASP) (143) in some sentinel countries such as the Philippines and Thailand (144), aimed at 690 collecting standardized and quality-assured epidemiological, clinical, microbiological and AMR 691 data. On top of these programs, WHO launched the Global AMR Surveillance System (GLASS) 692 in 2015 to foster national surveillance systems and enable standardized, comparable and 693 validated AMR data on priority human bacterial pathogens (145). Efforts are now underway to link 694 WHO GASP to GLASS. However, gonococcal AMR surveillance is still suboptimal or even lacking 695 in many locations, especially in LMICs, such as several parts of Asia, Central and Latin America, 696 Eastern Europe and Africa, which worryingly have the greatest incidence of gonorrhoea (3). 697 LMICs often have access to antimicrobials without prescription, have limited access to an optimal 698 treatment, lack the capacity needed to perform a laboratory diagnosis due to limited or non- 
existent quality-assured laboratories, microbiological and bioinformatics expertise or training,

700 insufficient availability and exorbitant prices of some reagents on top of a lack of funding, which

701 altogether compromises infection control.

702

High throughput sequencing approaches have proved invaluable over traditional molecular methods to identify AMR clones of bacterial pathogens, outbreaks, transmission networks and national and international spread among others $(28,29)$. Genomic surveillance efforts to capture the local and international spread of $N$. gonorrhoeae have resulted in several publications within the last decade involving high throughput sequence data of thousands of isolates from many locations across the world. The analysis of this data requires expertise, not always completely available, in bioinformatics, genomics, genetics, AMR, phylogenetics, epidemiology, etc. For lower-resourced settings, initiatives such as the NIHR Global Health Research Unit, Genomic

710 Surveillance of Antimicrobial Resistance (146) are essential to build genomic surveillance 711 capacity and provide the necessary microbiology and bioinformatics training for quality-assured genomic surveillance of AMR.

713 One of the strengths of genomic epidemiology is being able to compare new genomes with 714 existing data from a broader geographical level, which provides additional information on, e.g. if 715 new cases are part of a single clonal expansion or multiple introductions from outside a specific 716 location. To support this, Pathogenwatch calculates phylogenetic trees from a set of genomes 717 selected as collections. Currently, over 12,000 isolates of $N$. gonorrhoeae have been sequenced using high throughput approaches and publicly deposited on the ENA linked to a scientific

719 publication. We have quality-checked and assembled these data using a common pipeline and 720 we made it available through Pathogenwatch, with the aim of representing as much genomic 721 diversity of this pathogen as possible to serve as background for new analyses. These public 722 genomes are associated with at least 27 different scientific publications, and have been organized 723 in Pathogenwatch as individual collections (Additional file 1: Table S6). The clustering of strains 
examples in Figure S11), while differences in branch lengths may be attributed to the usage of different reconstruction methods.

727 The power of Pathogenwatch to investigate questions of public health concern is reflected in a case study (Figure 6). By selecting 1,142 azithromycin resistant strains from the public data in

729 Pathogenwatch in the context of a global collection (64), we observed one clone carrying $N$.

730 lactamica-like $m t r$ mosaic ('mosaic_2') in both the $m t r R$ promoter and $m t r D$ genes, likely resulting

731 from the same recombination event. Strong geographical structure was found in these

732 azithromycin resistant strains, with isolates from the USA (mostly from 2012-2016) clearly 733 differentiated from those from Australia (from 2017), which show a more clonal dispersion, likely

734 from a single main introduction to the country followed by a rapid spread. Interestingly, a sublineage of this Australian mtr mosaic-carrying clone seems to have also diverged after losing

736 the porB1b G120K and D121N mutations. It is important to note that the data from which these

737 inferences were derived was gathered from surveillance-based studies and outbreak

738 investigations, which may bias the observed global diversity of strains carrying this mosaic.

739 Phenotypic susceptibility data for azithromycin or epidemiological information were not available

740 for over half of these strains, thus impeding making further inferences. This reflects the need of

741 improving the submission of anonymized epidemiological and antimicrobial susceptibility data for

742 individual isolates rather than aggregated data to public repositories and/or as supplementary

743 information of the corresponding publications, as this is where the public data in Pathogenwatch

744 is coming from.

745 In this study, we have additionally gathered an advisory group of $N$. gonorrhoeae experts in 746 different fields such as AMR, microbiology, genetics, genomics, epidemiology and public health 747 who will consult and discuss current and future analytics to be included to address the global 748 public health needs of the community. We suggest this strategy as a role model for other 749 pathogens in this and other genomic surveillance platforms, so the end user, who may not have 750 full computational experience in some cases, can be confident that the analytics and databases 751 underlying this tool are appropriate, and can have access to all the results provided by 
752 Pathogenwatch through uploading the data via a web browser. We are aware that this is a

753 constantly moving field and analytics will be expanded and updated in the future. These updates

754 will be discussed within an advisory group to make sure they are useful in the field and the way

755 results are reported is of use to different profiles (microbiologists, epidemiologists, public health

756 professionals, etc.). Future analytics that are under discussion include the automatic submission

757 of new MLST, NG-STAR and NG-MAST STs and alleles to the corresponding servers, e.g.

758 PubMLST (48) and the automatic submission of data to public archives such as the ENA. Inter-

759 connectivity and comparability of results with PubMLST is of particular interest, as this database

760 has traditionally been the reference for Neisseria sequence typing and genomics and it is widely

761 used by the $N$. gonorrhoeae community. Plasmid and tetM/blaTEM subtyping as recently

762 described (147) will also be considered within the development roadmap of Pathogenwatch.

763 Including a separate library to automatically screen targets of potential interest for vaccine design

$764(148-150)$ as well as targets of new antibiotics currently in phase III clinical trials (i.e. zoliflodacin

765 (151) or gepotidacin (152)) can also be an interesting addition to the scheme. Regarding AMR,

766 new methods for phenotypic prediction using genetic data are continuously being reported (62,

767 153, 154), especially those based on machine learning algorithms (155), and will be considered

768 for future versions of the platform. The prediction of MIC values or ranges instead of SIR

769 categories will allow users to decide whether to use EUCAST (156) or CLSI (157) guidelines for

770 categorization.

\section{Conclusions}

773 In summary, we present a genomic surveillance platform adapted to $N$. gonorrhoeae, one of the

774 main public health priorities compromising the control of AMR infections, where decisions on

775 existing and updated databases and analytics as well as how results are reported will be

776 discussed with an advisory board of experts in different public health areas. This will allow

777 scientists from both higher or lower resourced settings with different capacities regarding high 
778 throughput sequencing, bioinformatics and data interpretation, to be able to use a reproducible

779 and quality-assured platform where analyse and contextualise genomic data resulting from the 780 investigation of treatment failures, outbreaks, transmission chains and networks at different

781 regional scales. This open access and reproducible platform constitutes one step further into an

782 international collaborative effort where countries can keep ownership of their data in line with 783 national STI and AMR surveillance and control programs while aligning with global strategies for

784 a joint action towards battling AMR N. gonorrhoeae.

785

786 List of abbreviations

787 AGSP: Australian Gonococcal Surveillance Programme

788 AMR: Antimicrobial Resistance

789 AZM: Azithromycin

790 CDC: Centers for Disease Control and Prevention

791 CFM: Cefixime

792 cgMLST: Core Genome Multi-Locus Sequence Typing

793 CIP: Ciprofloxacin

794 CLSI: Clinical Laboratory and Standards Institute

795 CRO: Ceftriaxone

796 ECOFF: Epidemiological Cut-Off

797 EGASP: Enhanced Gonococcal Antimicrobial Surveillance Programme

798 ENA: European Nucleotide Archive

799 ESCs: Extended Spectrum Cephalosporins

800 EUCAST: European Committee on Antimicrobial Susceptibility Testing

801 Euro-GASP: European Gonococcal Antimicrobial Surveillance Programme

802 FN: False Negative

803 FP: False Positive

804 GASP: Gonococcal Antimicrobial Surveillance Programme 
805 GISP: Gonococcal Isolate Surveillance Project

806 GRASP: Gonococcal Resistance to Antimicrobials Surveillance Programme

807 HIV: Human Immunodeficiency Virus

808 LMICs: Low and Middle-Income Countries

809 MIC: Minimum Inhibitory Concentration

810 MLST: Multi-Locus Sequence Typing

811 NG-MAST: N. gonorrhoeae Multi-Antigen Sequence Typing

812 NG-STAR: N. gonorrhoeae Sequence Typing for Antimicrobial Resistance

813 NPV: Negative Predictive Value

814 PEN: Benzylpenicillin

815 PPV: Positive Predictive Value

816 SNPs: Single Nucleotide Polymorphisms

817 ST: Sequence Type

818 STI: Sexually-Transmitted Infection

819 TET: Tetracycline

820 TN: True Negative

821 TP: True Positive

822 UK: United Kingdom

823 WGS: Whole Genome Sequencing

824 WHO: World Health Organization

825

826

827

828

829

830 


\section{Declarations}

832

\section{Ethics approval and consent to participate}

834 Not applicable.

835

836

\section{Consent for publication}

837 Not applicable.

838

\section{Availability of data and materials}

840 The assemblies included in the current version of the $N$. gonorrhoeae Pathogenwatch scheme

841 and used for the AMR benchmark analyses were generated from raw sequencing data stored in 842 the ENA. Project accession numbers are included in Additional File 1: Tables S1 and S6. The 843 generated assemblies can be downloaded from Pathogenwatch. The AMR library can be 844 accessed from: https://gitlab.com/cgps/pathogenwatch/amr-libraries/-/blob/master/485.toml. The 845 code to reproduce the figures and analyses in this manuscript can be found in 846 https://gitlab.com/cgps/pathogenwatch/publications/-/tree/master/ngonorrhoeae.

\section{Competing interests}

849 The authors declare that they have no competing interests.

850

\section{$851 \quad$ Funding}

852 Pathogenwatch is developed with support from Li Ka Shing Foundation (Big Data Institute, 853 University of Oxford) and Wellcome (099202). At the time of preparation of this manuscript, LSB 854 was supported by the Li Ka Shing Foundation (Big Data Institute, University of Oxford) and the 855 Centre for Genomic Pathogen Surveillance (CGPS, http://pathogensurveillance.net). At the time 856 of review and publication of this manuscript, LSB is funded by Plan GenT (CDEI-06/20-B), 857 Conselleria de Sanidad Universal y Salud Pública, Generalitat Valenciana (Valencia, Spain). DMA 858 is supported by the Li Ka Shing Foundation (Big Data Institute, University of Oxford) and the 
859 Centre for Genomic Pathogen Surveillance (CGPS). DMA and SA are supported by the National

860 Institute for Health Research (UK) Global Health Research Unit on Genomic Surveillance of AMR

861 (16_136_111). The department of MJC receives funding from the European Centre for Disease

862 Prevention and Control and the National Institute for Health Research (Health Protection

863 Research Unit) for gonococcal whole-genome sequencing. YHG is supported by the NIH/NIAID

864 grants R01 Al132606 and R01 Al153521. KCM is supported by the NSF GRFP grant

number DGE1745303. TDM is supported by the National Institute of Allergy and Infectious

866 Diseases at the National Institutes of Health [1 F32 Al145157-01]. WMS is a recipient of a Senior

867 Research Career Scientist Award from the Biomedical Laboratory Research and Development

868 Service of the Department of Veterans. Work on antibiotic resistance in his laboratory is supported

869 by $\mathrm{NIH}$ grants $\mathrm{R} 37 \mathrm{Al}-021150$ and R01 Al-147609. The content of this article is solely the responsibility of the authors and does not necessarily represent the official views of the

871 Department of Veterans Affairs, The National Institutes of Health or the United States

872 Government. The findings and conclusions in this article are those of the author(s) and do not

873 necessarily represent the official position of the Centers for Disease Control and Prevention. The

874 WHO Collaborating Centre for Gonorrhoea and other STIs represented by DG and MU receives

875 funding from the European Centre for Disease Prevention and Control and the World Health

876 Organization. This publication made use of the Neisseria Multi-Locus Sequence Typing website

877 (https://pubmlst.org/neisseria/) sited at the University of Oxford (48) and funded by Wellcome and

878 European Union.

879

880 Authors' contributions

881 DMA conceived the Pathogenwatch application. CY, RG, KA, BT, AU and DMA developed the 882 Pathogenwatch application. LSB and DMA contributed to the conception and design of the work. 883 CY and LSB generated, updated and benchmarked the N. gonorrhoeae AMR library. BT, CY, AU and LSB obtained, quality-checked and reassembled the raw data from the ENA. LSB revised the assembled data, obtained all metadata available from the corresponding scientific publications and created collections. LSB, CY and DMA analysed the data. LSB and DMA drafted the 
manuscript. LSB, DMA, CY, SA, KCM, TDM, DG, MJC, YHG, IM, BHR, WMS, GS, KT, TW, SRH

and MU contributed to the acquisition, technical and scientific interpretation and discussion of the and participated in virtual discussions. All authors read and approved the final manuscript.

\section{Acknowledgements}

We would like to thank MJC, YHG, IM, BHR, WMS, GS, KT, TW and MU for their support on the development of the $N$. gonorrhoeae Pathogenwatch scheme and the creation of the $N$. gonorrhoeae Pathogenwatch Scientific Steering Group.

\section{References}

899

1. World Health Organization (WHO). Global priority list of antibiotic-resistant bacteria to

900

901

902

903

904

905

906

907

908 guide research, discovery, and development of new antibiotics. http://www.who.int/medicines/publications/WHO-PPL-Short Summary 25FebET NM WHO.pdf. 2017.

2. Centers for Disease Prevention and Control. U.S. Department of Health and Human Services, Atlanta, GA. Antibiotic Resistance Threats in the United States, 2019. https://www.cdc.gov/drugresistance/pdf/threats-report/2019-ar-threats-report-508.pdf. 2019. 3. Rowley J, Vander Hoorn S, Korenromp E, Low N, Unemo M, Abu-Raddad LJ, et al. Chlamydia, gonorrhoea, trichomoniasis and syphilis: global prevalence and incidence estimates, 2016. Bull World Health Organ. 2019;97(8):548-62P.

909 4. World Health Organization (WHO). Report on global sexually transmitted infection surveillance 2018. http://apps.who.int/iris/bitstream/handle/10665/277258/9789241565691eng.pdf?ua=1. 2019.

5. Unemo M, Shafer WM. Antimicrobial resistance in Neisseria gonorrhoeae in the 21st century: past, evolution, and future. Clin Microbiol Rev. 2014;27(3):587-613.

6. Cole MJ, Spiteri G, Town K, Unemo M, Hoffmann S, Chisholm SA, et al. Risk factors for antimicrobial-resistant Neisseria gonorrhoeae in Europe. Sex Transm Dis. 2014;41(12):723-9. 7. Wi T, Lahra MM, Ndowa F, Bala M, Dillon JR, Ramon-Pardo P, et al. Antimicrobial resistance in Neisseria gonorrhoeae: Global surveillance and a call for international collaborative action. PLoS Med. 2017;14(7):e1002344.

8. Unemo M, Lahra MM, Cole M, Galarza P, Ndowa F, Martin I, et al. World Health Organization Global Gonococcal Antimicrobial Surveillance Program (WHO GASP): review of new data and evidence to inform international collaborative actions and research efforts. Sex Health. 2019;16(5):412-25.

9. Fifer H, Natarajan U, Jones L, Alexander S, Hughes G, Golparian D, et al. Failure of Dual Antimicrobial Therapy in Treatment of Gonorrhea. N Engl J Med. 2016;374(25):2504-6. 10. Jennison A, Whiley D, Lahra M, Graham R, Cole M, Hughes G, et al. Genetic relatedness of ceftriaxone-resistant and high-level azithromycin resistant Neisseria gonorrhoeae cases, United Kingdom and Australia, February to April 2018. . Euro Surveill. 
11. Lahra MM, Martin I, Demczuk W, Jennison AV, Lee KI, Nakayama SI, et al. Cooperative Recognition of Internationally Disseminated Ceftriaxone-Resistant Neisseria gonorrhoeae Strain. Emerg Infect Dis. 2018;24(4).

932 12. Fifer H, Saunders J, Soni S, Sadiq ST, FitzGerald M. 2018 UK national guideline for the management of infection with Neisseria gonorrhoeae. Int J STD AIDS. 2020;31(1):4-15. 13. Deguchi T, Yasuda M, Asano M, Tada K, Iwata H, Komeda H, et al. DNA gyrase mutations in quinolone-resistant clinical isolates of Neisseria gonorrhoeae. Antimicrob Agents Chemother. 1995;39(2):561-3.

14. Tanaka M, Takahashi K, Saika T, Kobayashi I, Ueno T, Kumazawa J. Development of fluoroquinolone resistance and mutations involving GyrA and ParC proteins among Neisseria gonorrhoeae isolates in Japan. J Urol. 1998;159(6):2215-9.

15. Harris SR, Cole MJ, Spiteri G, Sanchez-Buso L, Golparian D, Jacobsson S, et al. Public health surveillance of multidrug-resistant clones of Neisseria gonorrhoeae in Europe: a genomic survey. Lancet Infect Dis. 2018;18(7):758-68.

943 16. Spratt BG. Hybrid penicillin-binding proteins in penicillin-resistant strains of Neisseria 944 gonorrhoeae. Nature. 1988;332(6160):173-6.

945 17. Ohnishi M, Golparian D, Shimuta K, Saika T, Hoshina S, Iwasaku K, et al. Is Neisseria gonorrhoeae initiating a future era of untreatable gonorrhea?: detailed characterization of the first strain with high-level resistance to ceftriaxone. Antimicrob Agents Chemother. 2011;55(7):3538-45.

18. Grad YH, Harris SR, Kirkcaldy RD, Green AG, Marks DS, Bentley SD, et al. Genomic epidemiology of gonococcal resistance to extended spectrum cephalosporins, macrolides, and fluoroquinolones in the US, 2000-2013. J Infect Dis. 2016;214:1579-87.

19. Abrams AJ, Kirkcaldy RD, Pettus K, Fox JL, Kubin G, Trees DL. A Case of Decreased Susceptibility to Ceftriaxone in Neisseria gonorrhoeae in the Absence of a Mosaic PenicillinBinding Protein 2 (penA) Allele. Sex Transm Dis. 2017;44(8):492-4.

955 20. Lindberg R, Fredlund $\mathrm{H}$, Nicholas R, Unemo M. Neisseria gonorrhoeae isolates with reduced susceptibility to cefixime and ceftriaxone: association with genetic polymorphisms in penA, mtrR, porB1b, and ponA. Antimicrob Agents Chemother. 2007;51(6):2117-22. mutations cause cephalosporin resistance in clinical Neisseria gonorrhoeae isolates. Elife. 2020;9.

22. Unemo M, Golparian D, Sanchez-Buso L, Grad Y, Jacobsson S, Ohnishi M, et al. The novel 2016 WHO Neisseria gonorrhoeae reference strains for global quality assurance of laboratory investigations: phenotypic, genetic and reference genome characterization. $J$ Antimicrob Chemother. 2016;71(11):3096-108.

\section{Wadsworth CB, Arnold BJ, Sater MRA, Grad YH. Azithromycin Resistance through} Interspecific Acquisition of an Epistasis-Dependent Efflux Pump Component and Transcriptional Regulator in Neisseria gonorrhoeae. mBio. 2018;9(4).

24. Rouquette-Loughlin CE, Reimche JL, Balthazar JT, Dhulipala V, Gernert KM, Kersh EN, et al. Mechanistic Basis for Decreased Antimicrobial Susceptibility in a Clinical Isolate of Neisseria gonorrhoeae Possessing a Mosaic-Like mtr Efflux Pump Locus. mBio. 2018;9(6). 25. Ma KC, Mortimer TD, Duckett MA, Hicks AL, Wheeler NE, Sanchez-Buso L, et al. Increased power from conditional bacterial genome-wide association identifies macrolide resistance mutations in Neisseria gonorrhoeae. Nat Commun. 2020;11(1):5374.

26. Ma KC, Mortimer TD, Hicks AL, Wheeler NE, Sanchez-Buso L, Golparian D, et al. Adaptation to the cervical environment is associated with increased antibiotic susceptibility in Neisseria gonorrhoeae. Nat Commun. 2020;11(1):4126.

27. Unemo M, Dillon JA. Review and international recommendation of methods for typing Neisseria gonorrhoeae isolates and their implications for improved knowledge of gonococcal epidemiology, treatment, and biology. Clin Microbiol Rev. 2011;24(3):447-58.

28. Loman NJ, Pallen MJ. Twenty years of bacterial genome sequencing. Nat Rev Microbiol. 2015;13(12):787-94. 
29. Balloux F, Bronstad Brynildsrud O, van Dorp L, Shaw LP, Chen H, Harris KA, et al. From Theory to Practice: Translating Whole-Genome Sequencing (WGS) into the Clinic. Trends Microbiol. 2018;26(12):1035-48.

30. Boolchandani M, D'Souza AW, Dantas G. Sequencing-based methods and resources to study antimicrobial resistance. Nat Rev Genet. 2019;20(6):356-70.

31. Hendriksen RS, Bortolaia V, Tate H, Tyson GH, Aarestrup FM, McDermott PF. Using Genomics to Track Global Antimicrobial Resistance. Front Public Health. 2019;7:242.

32. React. https://reactjs.org/. Accessed 24 November 2020.

33. Material Design Lite. https://getmdl.io. Accessed 24 November 2020.

34. Phylocanvas. http://phylocanvas.org. Accessed 24 November 2020.

35. Leaflet. https://leafletjs.com/. Accessed 24 November 2020.

36. Sigma. http://sigmajs.org/. Accessed 24 November 2020.

37. Argimon S, Abudahab K, Goater RJ, Fedosejev A, Bhai J, Glasner C, et al. Microreact: visualizing and sharing data for genomic epidemiology and phylogeography. Microb Genom. 2016;2(11):e000093.

38. Page AJ, Cummins CA, Hunt M, Wong VK, Reuter S, Holden MT, et al. Roary: rapid large-scale prokaryote pan genome analysis. Bioinformatics. 2015;31(22):3691-3.

39. Centre for Genomic Pathogen Surveillance (CGPS). Pathogenwatch technical descriptions. Core assignment. https://cgps.gitbook.io/pathogenwatch/technicaldescriptions/core-genome-tree/core-assignment. Accessed 24 November 2020.

40. Bankevich A, Nurk S, Antipov D, Gurevich AA, Dvorkin M, Kulikov AS, et al. SPAdes: a new genome assembly algorithm and its applications to single-cell sequencing. J Comput Biol. 2012;19(5):455-77.

41. Centre for Genomic Pathogen Surveillance (CGPS). Pathogenwatch technical descriptions. Short read assembly. https://cgps.gitbook.io/pathogenwatch/technicaldescriptions/short-read-assembly. Accessed 24 November 2020.

42. CGPS. Pathogenwatch technical descriptions. Speciator. https://cgps.gitbook.io/pathogenwatch/technical-descriptions/species-assignment/speciator. Accessed 24 November 2020.

43. Centre for Genomic Pathogen Surveillance (CGPS). Pathogenwatch technical descriptions. Core filter. https://cgps.gitbook.io/pathogenwatch/technical-descriptions/coregenome-tree/core-filter. Accessed 24 November 2020.

44. Centre for Genomic Pathogen Surveillance (CGPS). Pathogenwatch technical descriptions. Tree construction. https://cgps.gitbook.io/pathogenwatch/technicaldescriptions/core-genome-tree/tree-construction. Accessed 24 November 2020.

45. Bennett JS, Jolley KA, Sparling PF, Saunders NJ, Hart CA, Feavers IM, et al. Species status of Neisseria gonorrhoeae: evolutionary and epidemiological inferences from multilocus sequence typing. BMC Biol. 2007;5:35.

46. Harrison OB, Cehovin A, Skett J, Jolley KA, Massari P, Genco CA, et al. Neisseria gonorrhoeae Population Genomics: Use of the Gonococcal Core Genome to Improve Surveillance of Antimicrobial Resistance. J Infect Dis. 2020;222(11):1816-25.

47. PubMLST. Neisseria Multi-Locus Sequence Typing website. https://pubmlst.org/neisserial. Accessed 24 November 2020.

48. Jolley KA, Bray JE, Maiden MCJ. Open-access bacterial population genomics: BIGSdb software, the PubMLST.org website and their applications. Wellcome Open Res. 2018;3:124.

49. Martin IM, Ison CA, Aanensen DM, Fenton KA, Spratt BG. Rapid sequence-based identification of gonococcal transmission clusters in a large metropolitan area. J Infect Dis. 2004;189(8):1497-505.

50. NG-MAST. Neisseria gonorrhoeae Multi-Antigen Sequence Typing database. http://www.ng-mast.net/. Accessed 24 November 2020.

51. Demczuk W, Sidhu S, Unemo M, Whiley DM, Allen VG, Dillon JR, et al. Neisseria gonorrhoeae Sequence Typing for Antimicrobial Resistance, a Novel Antimicrobial Resistance Multilocus Typing Scheme for Tracking Global Dissemination of N. gonorrhoeae Strains. J Clin Microbiol. 2017;55(5):1454-68. 
52. NG-STAR. Neisseria gonorrhoeae Sequence Typing for Antimicrobial Resistance. https://ngstar.canada.cal. Accessed 24 November 2020.

53. Kwong JC, Gonçalves da Silva A, Dyet K, Williamson DA, Stinear TP, Howden BP, et al. NGMASTER: in silico Multi-Antigen Sequence Typing for Neisseria gonorrhoeae. Microb Genom. 2016;2(8):e000076.

54. Centre for Genomic Pathogen Surveillance (CGPS). Pathogenwatch technical descriptions. MLST. https://cgps.gitbook.io/pathogenwatch/technical-descriptions/typingmethods/mlst. Accessed 24 November 2020.

55. Centre for Genomic Pathogen Surveillance (CGPS). Pathogenwatch technical descriptions. cgMLST clustering. https://cgps.gitbook.io/pathogenwatch/technicaldescriptions/cgmlst-clusters. Accessed 24 November 2020.

56. Sibson R. SLINK: An optimally efficient algorithm for the single-link cluster method. The Computer Journal. 1973;16(1):30-4.

57. Centre for Genomic Pathogen Surveillance (CGPS). Pathogenwatch technical descriptions. Pathogenwatch AMR. https://cgps.gitbook.io/pathogenwatch/technicaldescriptions/antimicrobial-resistance-prediction/pw-amr. Accessed 24 November 2020. 58. Chisholm SA, Wilson J, Alexander S, Tripodo F, Al-Shahib A, Schaefer U, et al. An outbreak of high-level azithromycin resistant Neisseria gonorrhoeae in England. Sex Transm Infect. 2015;0:1-3.

59. Golparian D, Harris SR, Sanchez-Buso L, Hoffmann S, Shafer WM, Bentley SD, et al. Genomic evolution of Neisseria gonorrhoeae since the preantibiotic era (1928-2013): antimicrobial use/misuse selects for resistance and drives evolution. BMC Genomics. 2020;21(1):116.

60. Demczuk W, Lynch T, Martin I, Van Domselaar G, Graham M, Bharat A, et al. Wholegenome phylogenomic heterogeneity of Neisseria gonorrhoeae isolates with decreased cephalosporin susceptibility collected in Canada between 1989 and 2013. J Clin Microbiol. 2015;53(1):191-200.

61. Demczuk W, Martin I, Peterson S, Bharat A, Van Domselaar G, Graham M, et al. Genomic Epidemiology and Molecular Resistance Mechanisms of Azithromycin-Resistant Neisseria gonorrhoeae in Canada from 1997 to 2014. J Clin Microbiol. 2016;54(5):1304-13. 62. Eyre DW, De Silva D, Cole K, Peters J, Cole MJ, Grad YH, et al. WGS to predict antibiotic MICs for Neisseria gonorrhoeae. J Antimicrob Chemother. 2017;72(7):1937-47. 63. Fifer H, Cole M, Hughes G, Padfield S, Smolarchuk C, Woodford N, et al. Sustained transmission of high-level azithromycin-resistant Neisseria gonorrhoeae in England: an observational study. Lancet Infect Dis. 2018;18(5):573-81.

64. Sanchez-Buso L, Golparian D, Corander J, Grad YH, Ohnishi M, Flemming R, et al. The impact of antimicrobials on gonococcal evolution. Nat Microbiol. 2019.

65. Grad YH, Kirkcaldy RD, Trees D, Dordel J, Harris SR, Goldstein E, et al. Genomic epidemiology of Neisseria gonorrhoeae with reduced susceptibility to cefixime in the USA: a retrospective observational study. Lancet Infect Dis. 2014;14(3):220-6.

66. Jacobsson S, Golparian D, Cole M, Spiteri G, Martin I, Bergheim T, et al. WGS analysis and molecular resistance mechanisms of azithromycin-resistant (MIC $>2 \mathrm{mg} / \mathrm{L}$ ) Neisseria gonorrhoeae isolates in Europe from 2009 to 2014. J Antimicrob Chemother. 2016.

67. Lee RS, Seemann T, Heffernan H, Kwong JC, Goncalves da Silva A, Carter GP, et al. Genomic epidemiology and antimicrobial resistance of Neisseria gonorrhoeae in New Zealand. J Antimicrob Chemother. 2018;73(2):353-64.

68. Town K, Harris S, Sanchez-Buso L, Cole MJ, Pitt R, Fifer H, et al. Genomic and Phenotypic Variability in Neisseria gonorrhoeae Antimicrobial Susceptibility, England. Emerg Infect Dis. 2020;26(3):505-15.

69. Yahara K, Nakayama SI, Shimuta K, Lee KI, Morita M, Kawahata T, et al. Genomic surveillance of Neisseria gonorrhoeae to investigate the distribution and evolution of antimicrobial-resistance determinants and lineages. Microb Genom. 2018;4(8).

70. Kwong JC, Chow EPF, Stevens K, Stinear TP, Seemann T, Fairley CK, et al. Wholegenome sequencing reveals transmission of gonococcal antibiotic resistance among men who have sex with men: an observational study. Sex Transm Infect. 2018;94(2):151-7. 
1091

1092

1093

1094

1095

1096

1097

1098

1099

1100

1101

1102

1103

1104

1105

1106

1107

1108

1109

1110

1111

1112

1113

1114

1115

1116

1117

1118

1119

1120

1121

1122

1123

1124

1125

1126

1127

1128

1129

1130

1131

1132

1133

1134

1135

1136

1137

1138

1139

1140

1141

1142

1143

1144

1145
71. European Committee on Antimicrobial Susceptibility Testing (EUCAST). Breakpoint tables for interpretation of MICs and zone diameters. Version 9.0.

https://www.eucast.org/fileadmin/src/media/PDFs/EUCAST files/Breakpoint tables/v 9.0 Brea kpoint Tables.pdf. Accessed 24 November 2020.

72. Stevenson M, Nunes T, Heuer C, Marshall J, Sanchez J, Thornton R, et al. epiR: Tools for the Analysis of Epidemiological Data. R package version 1.0-14. https://CRAN.Rproject.org/package=epiR. 2020.

73. Carver T, Harris SR, Berriman M, Parkhill J, McQuillan JA. Artemis: an integrated platform for visualization and analysis of high-throughput sequence-based experimental data. Bioinformatics. 2012;28(4):464-9.

74. Chisholm SA, Dave J, Ison CA. High-level azithromycin resistance occurs in Neisseria gonorrhoeae as a result of a single point mutation in the 23S rRNA genes. Antimicrob Agents Chemother. 2010;54(9):3812-6.

75. Underwood A. Gobal Health Research Unit (GHRU) assembly pipeline.

https://gitlab.com/cgps/ghru/pipelines/assembly. Accessed 24 November 2020.

76. Bioinformatics B. FastQC. https://bioinformatics.babraham.ac.uk/projects/fastqc/. Accessed 24 November 2020.

77. Bolger AM, Lohse M, Usadel B. Trimmomatic: a flexible trimmer for Illumina sequence data. Bioinformatics. 2014;30(15):2114-20.

78. Song L, Florea L, Langmead B. Lighter: fast and memory-efficient sequencing error correction without counting. Genome Biol. 2014;15(11):509.

79. Low AJ, Koziol AG, Manninger PA, Blais B, Carrillo CD. ConFindr: rapid detection of intraspecies and cross-species contamination in bacterial whole-genome sequence data. PeerJ. 2019;7:e6995.

80. Ondov BD, Treangen TJ, Melsted P, Mallonee AB, Bergman NH, Koren S, et al. Mash: fast genome and metagenome distance estimation using MinHash. Genome Biol. 2016;17(1):132.

81. Li H. Seqtk. https://github.com/lh3/seqtk. Accessed 24 November 2020.

82. Magoc T, Salzberg SL. FLASH: fast length adjustment of short reads to improve genome assemblies. Bioinformatics. 2011;27(21):2957-63.

83. Gurevich A, Saveliev V, Vyahhi N, Tesler G. QUAST: quality assessment tool for genome assemblies. Bioinformatics. 2013;29(8):1072-5.

84. Underwood A. Bactinspector. https://gitlab.com/antunderwood/bactinspector. Accessed 24 November 2020.

85. Underwood A. Qualifyr. https://gitlab.com/cgps/qualifyr. Accessed 24 November 2020.

86. European Nucleotide Archive API portal. https://www.ebi.ac.uk/ena/portal/api/. Accessed 24 November 2020.

87. Sánchez-Busó L. N. gonorrhoeae Pathogenwatch video demo.

https://vimeo.com/375402189. 2020

88. Centre for Genomic Pathogen Surveillance (CGPS). Pathogenwatch collection.

Sánchez-Busó et al. (2019). https://pathogen.watch/collection/9stz0m94hin7-sanchez-buso-etal-2019.

89. Page A, Taylor B, Keane J. Multilocus sequence typing by blast from de novo assemblies against PubMLST. Journal of Open Source Software. 2016;1(8).

90. $\mathrm{Ng} \mathrm{LK}$, Martin I, Liu G, Bryden L. Mutation in 23S rRNA associated with macrolide resistance in Neisseria gonorrhoeae. Antimicrob Agents Chemother. 2002;46(9):3020-5.

91. Roberts MC, Chung WO, Roe D, Xia M, Marquez C, Borthagaray G, et al. Erythromycinresistant Neisseria gonorrhoeae and oral commensal Neisseria spp. carry known rRNA methylase genes. Antimicrob Agents Chemother. 1999;43(6):1367-72.

92. Cousin S, Jr., Whittington WL, Roberts MC. Acquired macrolide resistance genes in pathogenic Neisseria spp. isolated between 1940 and 1987. Antimicrob Agents Chemother. 2003;47(12):3877-80.

93. Luna VA, Cousin S, Jr., Whittington WL, Roberts MC. Identification of the conjugative mef gene in clinical Acinetobacter junii and Neisseria gonorrhoeae isolates. Antimicrob Agents Chemother. 2000;44(9):2503-6. 
1146 94. Rouquette-Loughlin CE, Balthazar JT, Shafer WM. Characterization of the MacA-MacB efflux system in Neisseria gonorrhoeae. J Antimicrob Chemother. 2005;56(5):856-60. 95. Veal WL, Nicholas RA, Shafer WM. Overexpression of the MtrC-MtrD-MtrE efflux pump due to an mtrR mutation is required for chromosomally mediated penicillin resistance in Neisseria gonorrhoeae. J Bacteriol. 2002;184(20):5619-24. the $1 \mathrm{bp}$ deletion in the mtrR promoter in Neisseria gonorrhoeae. J Antimicrob Chemother. 2003;51(1):131-3.

97. Warner DM, Shafer WM, Jerse AE. Clinically relevant mutations that cause derepression of the Neisseria gonorrhoeae MtrC-MtrD-MtrE Efflux pump system confer different levels of antimicrobial resistance and in vivo fitness. Mol Microbiol. 2008;70(2):462-78.

98. Shafer WM, Balthazar JT, Hagman KE, Morse SA. Missense mutations that alter the DNA-binding domain of the MtrR protein occur frequently in rectal isolates of Neisseria gonorrhoeae that are resistant to faecal lipids. Microbiology. 1995;141 ( Pt 4):907-11.

99. Tomberg J, Unemo M, Davies C, Nicholas RA. Molecular and structural analysis of mosaic variants of penicillin-binding protein 2 conferring decreased susceptibility to expandedspectrum cephalosporins in Neisseria gonorrhoeae: role of epistatic mutations. Biochemistry. 2010;49(37):8062-70.

100. Tomberg J, Unemo M, Ohnishi M, Davies C, Nicholas RA. Identification of amino acids conferring high-level resistance to expanded-spectrum cephalosporins in the penA gene from Neisseria gonorrhoeae strain H041. Antimicrob Agents Chemother. 2013;57(7):3029-36. 101. Unemo M, Golparian D, Nicholas R, Ohnishi M, Gallay A, Sednaoui P. High-level cefixime- and ceftriaxone-resistant Neisseria gonorrhoeae in France: novel penA mosaic allele in a successful international clone causes treatment failure. Antimicrob Agents Chemother. 2012;56(3):1273-80. 102. Belland RJ, Morrison SG, Ison C, Huang WM. Neisseria gonorrhoeae acquires mutations in analogous regions of gyrA and parC in fluoroquinolone-resistant isolates. Mol Microbiol. 1994;14(2):371-80. pump of Neisseria gonorrhoeae and Neisseria meningitidis recognizes antimicrobial cationic compounds. J Bacteriol. 2003;185(3):1101-6.

104. Lindback E, Rahman M, Jalal S, Wretlind B. Mutations in gyrA, gyrB, parC, and parE in quinolone-resistant strains of Neisseria gonorrhoeae. APMIS. 2002;110(9):651-7.

105. Hu M, Nandi S, Davies C, Nicholas RA. High-level chromosomally mediated tetracycline resistance in Neisseria gonorrhoeae results from a point mutation in the rps J gene encoding ribosomal protein $\mathrm{S} 10$ in combination with the $\mathrm{mtrR}$ and penB resistance determinants. Antimicrob Agents Chemother. 2005;49(10):4327-34.

106. Morse SA, Johnson SR, Biddle JW, Roberts MC. High-level tetracycline resistance in Neisseria gonorrhoeae is result of acquisition of streptococcal tetM determinant. Antimicrob Agents Chemother. 1986;30(5):664-70.

107. Ashford WA, Golash RG, Hemming VG. Penicillinase-producing Neisseria gonorrhoeae. Lancet. 1976;2(7987):657-8.

108. Ropp PA, Hu M, Olesky M, Nicholas RA. Mutations in ponA, the gene encoding penicillin-binding protein 1 , and a novel locus, penC, are required for high-level chromosomally mediated penicillin resistance in Neisseria gonorrhoeae. Antimicrob Agents Chemother. 2002;46(3):769-77.

109. Olesky M, Hobbs M, Nicholas RA. Identification and analysis of amino acid mutations in porin IB that mediate intermediate-level resistance to penicillin and tetracycline in Neisseria gonorrhoeae. Antimicrob Agents Chemother. 2002;46(9):2811-20.

110. Bilgin N, Richter AA, Ehrenberg M, Dahlberg AE, Kurland CG. Ribosomal RNA and protein mutants resistant to spectinomycin. EMBO J. 1990;9(3):735-9.

111. Unemo M, Golparian D, Skogen V, Olsen AO, Moi H, Syversen G, et al. Neisseria gonorrhoeae strain with high-level resistance to spectinomycin due to a novel resistance mechanism (mutated ribosomal protein S5) verified in Norway. Antimicrob Agents Chemother. 1200 2013;57(2):1057-61. 
112. Fiebelkorn KR, Crawford SA, Jorgensen JH. Mutations in folP associated with elevated sulfonamide MICs for Neisseria meningitidis clinical isolates from five continents. Antimicrob Agents Chemother. 2005;49(2):536-40.

113. Zhao S, Duncan M, Tomberg J, Davies C, Unemo M, Nicholas RA. Genetics of chromosomally mediated intermediate resistance to ceftriaxone and cefixime in Neisseria gonorrhoeae. Antimicrob Agents Chemother. 2009;53(9):3744-51.

114. Ison CA, Golparian D, Saunders P, Chisholm S, Unemo M. Evolution of Neisseria gonorrhoeae is a continuing challenge for molecular detection of gonorrhoea: false negative gonococcal porA mutants are spreading internationally. Sex Transm Infect. 2013;89(3):197-201. 115. Kersh EN, Allen V, Ransom E, Schmerer M, Cyr S, Workowski K, et al. Rationale for a Neisseria gonorrhoeae Susceptible-only Interpretive Breakpoint for Azithromycin. Clin Infect Dis. 2020;70(5):798-804.

116. Cole MJ, Tan W, Fifer H, Brittain C, Duley L, Hepburn T, et al. Gentamicin, azithromycin and ceftriaxone in the treatment of gonorrhoea: the relationship between antibiotic MIC and clinical outcome. J Antimicrob Chemother. 2020;75(2):449-57.

117. Town K, Field N, Harris SR, Sanchez-Buso L, Cole MJ, Pitt R, et al. Phylogenomic analysis of Neisseria gonorrhoeae transmission to assess sexual mixing and HIV transmission risk in England: a cross-sectional, observational, whole-genome sequencing study. Lancet Infect Dis. 2020;20(4):478-86.

118. Williamson DA, Chow EPF, Gorrie CL, Seemann T, Ingle DJ, Higgins N, et al. Bridging of Neisseria gonorrhoeae lineages across sexual networks in the HIV pre-exposure prophylaxis era. Nat Commun. 2019;10(1):3988.

119. De Silva D, Peters J, Cole K, Cole MJ, Cresswell F, Dean G, et al. Whole-genome sequencing to determine transmission of Neisseria gonorrhoeae: an observational study. Lancet Infect Dis. 2016;16(11):1295-303.

120. Mortimer TD, Pathela P, Crawley A, Rakeman JL, Lin Y, Harris SR, et al. The distribution and spread of susceptible and resistant Neisseria gonorrhoeae across demographic groups in a major metropolitan center. Clin Infect Dis. 2020.

121. Alfsnes K, Eldholm V, Olsen AO, Brynildsrud OB, Bohlin J, Steinbakk M, et al. Genomic epidemiology and population structure of Neisseria gonorrhoeae in Norway, 2016-2017. Microb Genom. 2020.

122. Thomas JC, Seby S, Abrams AJ, Cartee J, Lucking S, Vidyaprakash E, et al. Evidence of Recent Genomic Evolution in Gonococcal Strains With Decreased Susceptibility to Cephalosporins or Azithromycin in the United States, 2014-2016. J Infect Dis. 2019;220(2):294305.

123. Schmerer MW, Abrams AJ, Seby S, Thomas JCt, Cartee J, Lucking S, et al. Genomic Characterization of Neisseria gonorrhoeae Strains from 2016 U.S. Sentinel Surveillance Displaying Reduced Susceptibility to Azithromycin. Antimicrob Agents Chemother. 2020;64(5). 124. Lan PT, Golparian D, Ringlander J, Van Hung L, Van Thuong N, Unemo M. Genomic analysis and antimicrobial resistance of Neisseria gonorrhoeae isolates from Vietnam in 2011 and 2015-16. J Antimicrob Chemother. 2020;75(6):1432-8.

125. Didelot X, Dordel J, Whittles LK, Collins C, Bilek N, Bishop CJ, et al. Genomic Analysis and Comparison of Two Gonorrhea Outbreaks. MBio. 2016;7(3).

126. Osnes MN, Didelot X, Korne-Elenbaas J, Alfsnes K, Brynildsrud OB, Syversen G, et al. Sudden emergence of a Neisseria gonorrhoeae clade with reduced susceptibility to extendedspectrum cephalosporins, Norway. Microb Genom. 2020.

127. Cehovin A, Harrison OB, Lewis SB, Ward PN, Ngetsa C, Graham SM, et al. Identification of Novel Neisseria gonorrhoeae Lineages Harboring Resistance Plasmids in Coastal Kenya. J Infect Dis. 2018;218(5):801-8.

128. Buckley C, Forde BM, Trembizki E, Lahra MM, Beatson SA, Whiley DM. Use of whole genome sequencing to investigate an increase in Neisseria gonorrhoeae infection among women in urban areas of Australia. Sci Rep. 2018;8(1):1503.

129. Ryan L, Golparian D, Fennelly N, Rose L, Walsh P, Lawlor B, et al. Antimicrobial resistance and molecular epidemiology using whole-genome sequencing of Neisseria 
gonorrhoeae in Ireland, 2014-2016: focus on extended-spectrum cephalosporins and azithromycin. Eur J Clin Microbiol Infect Dis. 2018;37(9):1661-72.

130. Wind CM, de Vries E, Schim van der Loeff MF, van Rooijen MS, van Dam AP, Demczuk WHB, et al. Decreased Azithromycin Susceptibility of Neisseria gonorrhoeae Isolates in Patients Recently Treated with Azithromycin. Clin Infect Dis. 2017;65(1):37-45.

131. Ezewudo MN, Joseph SJ, Castillo-Ramirez S, Dean D, Del Rio C, Didelot X, et al. Population structure of Neisseria gonorrhoeae based on whole genome data and its relationship with antibiotic resistance. PeerJ. 2015;3:e806.

132. Unemo M, Golparian D, Eyre DW. Antimicrobial Resistance in Neisseria gonorrhoeae and Treatment of Gonorrhea. Methods Mol Biol. 2019;1997:37-58.

133. Mortimer TD, Grad YH. Applications of genomics to slow the spread of multidrugresistant Neisseria gonorrhoeae. Ann N Y Acad Sci. 2019;1435(1):93-109.

134. Gernert KM, Seby S, Schmerer MW, Thomas JCt, Pham CD, Cyr SS, et al. Azithromycin susceptibility of Neisseria gonorrhoeae in the USA in 2017: a genomic analysis of surveillance data. Lancet Microbe. 2020;1(4):e154-e64.

135. Centre for Genomic Pathogen Surveillance (CGPS). Pathogenwatch collection. AZM resistant gonococcus in global context. https://pathogen.watch/collection/8mk1i3e55olu-azmresistant-gonococcus-in-global-context. Accessed 24 November 2020.

136. Wick RR, Holt KE. Benchmarking of long-read assemblers for prokaryote whole genome sequencing. F1000Res. 2019;8:2138.

137. Souvorov A, Agarwala R, Lipman DJ. SKESA: strategic k-mer extension for scrupulous assemblies. Genome Biol. 2018;19(1):153.

138. Spiteri G, Cole M, Unemo M, Hoffmann S, Ison C, van de Laar M. The European Gonococcal Antimicrobial Surveillance Programme (Euro-GASP)--a sentinel approach in the European Union (EU)/European Economic Area (EEA). Sex Transm Infect. 2013;89 Suppl 4:iv16-8.

139. Schwarcz SK, Zenilman JM, Schnell D, Knapp JS, Hook EW, 3rd, Thompson S, et al. National surveillance of antimicrobial resistance in Neisseria gonorrhoeae. The Gonococcal Isolate Surveillance Project. JAMA. 1990;264(11):1413-7.

140. Public Health Agency of Canada National Microbiology Laboratory. National surveillance of antimicrobial susceptibilities of Neisseria gonorrhoeae. Annual summary 2017.

https://www.canada.ca/en/public-health/services/publications/drugs-health-products/nationalsurveillance-antimicrobial-susceptibilities-neisseria-gonorrhoeae-annual-summary-2017.html. 2017.

141. Lahra MM, Enriquez R, George CRR. Australian Gonococcal Surveillance Programme Annual Report, 2017. Commun Dis Intell (2018). 2019;43.

142. Paine TC, Fenton KA, Herring A, Turner A, Ison C, Martin I, et al. GRASP: a new national sentinel surveillance initiative for monitoring gonococcal antimicrobial resistance in England and Wales. Sex Transm Infect. 2001;77(6):398-401.

143. Weston EJ, Wi T, Papp J. Strengthening Global Surveillance for Antimicrobial DrugResistant Neisseria gonorrhoeae through the Enhanced Gonococcal Antimicrobial Surveillance Program. Emerg Infect Dis. 2017;23(13).

144. Sirivongrangson P, Girdthep N, Sukwicha W, Buasakul P, Tongtoyai J, Weston E, et al. The first year of the global Enhanced Gonococcal Antimicrobial Surveillance Programme (EGASP) in Bangkok, Thailand, 2015-2016. PLoS One. 2018;13(11):e0206419.

145. Seale AC, Gordon NC, Islam J, Peacock SJ, Scott JAG. AMR Surveillance in low and middle-income settings - A roadmap for participation in the Global Antimicrobial Surveillance System (GLASS). Wellcome Open Res. 2017;2:92.

146. Global Health Research Unit (GHRU), Genomic Surveillance of Antimicrobial Resistance. https://ghru.pathogensurveillance.net/. Accessed 24 November 2020.

147. Cehovin A, Jolley KA, Maiden MCJ, Harrison OB, Tang CM. Association of Neisseria gonorrhoeae Plasmids With Distinct Lineages and The Economic Status of Their Country of Origin. J Infect Dis. 2020;222(11):1826-36. 
148. Gottlieb SL, Ndowa F, Hook EW, 3rd, Deal C, Bachmann L, Abu-Raddad L, et al. Gonococcal vaccines: Public health value and preferred product characteristics; report of a WHO global stakeholder consultation, January 2019. Vaccine. 2020.

149. Marjuki H, Topaz N, Joseph SJ, Gernert KM, Kersh EN, Antimicrobial-Resistant Neisseria gonorrhoeae Working Group, et al. Genetic Similarity of Gonococcal Homologs to Meningococcal Outer Membrane Proteins of Serogroup B Vaccine. mBio. 2019;10(5). 150. Russell MW, Jerse AE, Gray-Owen SD. Progress Toward a Gonococcal Vaccine: The Way Forward. Front Immunol. 2019;10:2417.

151. Taylor SN, Marrazzo J, Batteiger BE, Hook EW, 3rd, Sena AC, Long J, et al. SingleDose Zoliflodacin (ETX0914) for Treatment of Urogenital Gonorrhea. N Engl J Med. 2018;379(19):1835-45.

152. Taylor SN, Morris DH, Avery AK, Workowski KA, Batteiger BE, Tiffany CA, et al. Gepotidacin for the Treatment of Uncomplicated Urogenital Gonorrhea: A Phase 2, Randomized, Dose-Ranging, Single-Oral Dose Evaluation. Clin Infect Dis. 2018;67(4):504-12. 153. Institute of Medicine (US) Forum on Microbial Threats. Antibiotic Resistance: Implications for Global Health and Novel Intervention Strategies: Workshop Summary. The National Academies Collection: Reports funded by National Institutes of Health. Washington (DC)2010.

154. Demczuk W, Martin I, Sawatzky P, Allen V, Lefebvre B, Hoang L, et al. Equations To Predict Antimicrobial MICs in Neisseria gonorrhoeae Using Molecular Antimicrobial Resistance Determinants. Antimicrob Agents Chemother. 2020;64(3).

155. Hicks AL, Wheeler N, Sanchez-Buso L, Rakeman JL, Harris SR, Grad YH. Evaluation of parameters affecting performance and reliability of machine learning-based antibiotic susceptibility testing from whole genome sequencing data. PLoS Comput Biol. 2019;15(9):e1007349.

156. European Committee on Antimicrobial Susceptibility Testing (EUCAST). EUCAST clinical breakpoints. https://eucast.org/clinical breakpoints/. Accessed 2nd December 2020. 157. Clinical Laboratory and Standards Institute (CLSI) guidelines. https://clsi.org/. Accessed 2nd December 2020. 